\title{
Spiders (Arachnida: Aranei) of Stavropol Province, Russia
}

\section{Пауки (Arachnida: Aranei) Ставропольского края, Россия}

\author{
Alexander V. Ponomarev*, Sergey K. Alekseev**, \\ Vladislav O. Kozminykh***, Vladimir Yu. Shmatko* \\ А.В. Пономарёв*, С.К. Алексеев**, В.О. Козьминых***, \\ B.Ю. Шматко*
}

\footnotetext{
* Institute of Arid Zones, Southern Scientific Centre, Russian Academy of Sciences, Chekhov Str., 41, Rostov-on-Don 344006 Russia. Email: ponomarev1952@mail.ru

* Институт аридных зон, Южный научный центр РАН, пр. Чехова, 41, Ростов-на-Дону 344006 Россия.

** Ecological club “Stenus”, Staroobryadcheski per., 4, Kaluga 248600 Russia. E-mail: stenus@yandex.ru

** Экологический клуб “Stenus”, пер. Старообрядческий, 4, Калуга 248600 Россия.

*** Perm State Humanitarian Pedagogical University, Sibirskaya Str., 24, Perm 614990 Russia. E-mail: kvoncstu@yahoo.com

*** Пермский государственный гуманитарно-педагогический университет, ул. Сибирская, 24, Пермь 614990 Россия.
}

KEY WORDS: spiders, annotated checklist, Stavropol Province, Russia.

КЛЮЧЕВЫЕ СЛОВА: пауки, аннотированный список, Ставропольский край, Россия.

ABSTRACT. An annotated checklist of spiders recorded from Stavropol Province, based on literaturebased and new data, is provided. The checklist comprises 265 spider species of 26 families. Of them, 154 species have been recorded from Stavropol Province for the first time. One species (Zodarion rubidium Simon, 1914) represents a new record to the spider fauna of Russia. Eight species are illustrated.

РЕЗЮМЕ. Приведён список пауков, выявленных в Ставропольском крае (по литературным и собственным данным): 265 вида, 26 семейств. Из них 154 вида впервые отмечаются на территории Ставропольского края. Один вид (Zodarion rubidium Simon, 1914) является новым для фауны пауков России. Восемь видов проиллюстрированы.

\section{Introduction}

The spider fauna of Stavropol Province, in its present borders as a federal subject, is the least studied one among other regional faunas of southern Russia. Most of the spider species hitherto recorded from Stavropol Province are also known from Karachay-Cherkess Republic, which now is a separate federal subject. For example, A.V. Tanasevitch [Tanasevitch, 1987, 1990] listed 12 species of Linyphiidae for Stavropol Province, whereas his list for the entire Caucasus comprised around 160 species. The first study concerning spiders specifically devoted to Stavropol Province was published only recently [Logunov, Penney, 2004]. The latter study provides records of 35 species of 12 spider families collected near Beshpagir Village. Later on, some short faunistic reports [Ponomarev et al., 2006;
Khanov, Ponomarev, 2006] were published, providing additional spider records for Stavropol Province. In this paper, we aim at assembling all the previously published records along with newly collected data to provide an updated checklist of spiders from Stavropol Province.

\section{Material and methods}

The material used in the present study was collected by Sergey Alexeev (hereafter abbreviated as S.A.) from Arzgirsk and Neftekumsk Districts of Stavropol Province, by V.O. Kozminykh (V.K.) in the vicinity of Izobilnyi, and by T.V. Khanov (T.Kh.) and E. Khomchenko (E.Kh) in lowland areas of Stavropol Province. Additionally, we have examined some materials kindly shared with us by A.A. Zotov (A.Z.), P.P. Ivliev (P.I.), S.V. Pushkin (S.P.), A.E. Rudaikov (A.R.), E.N. Terskov (E.T.), Yu.A. Manelov (Yu.M.).

Scanning Electron Microscope images of specimens used in this study were taken by K.V. Dvadnenko at the interdisciplinary analytical laboratory of the Institute of Arid Zones (Rostov-on-Don). Digital images were taken by means of MIKMED-6 using digital camera SONY NEX-C3 16.2mp and adapters (MFN-12). All the examined material is deposited at the private collection of A.V. Ponomarev.

The following abbreviation is used: s.l. - same locality.

The localities mentioned in this study and their geographical coordinates (Map 1) are as follows:

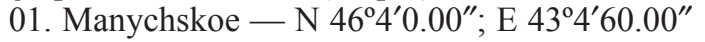

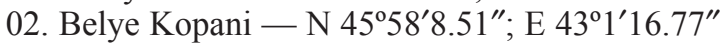




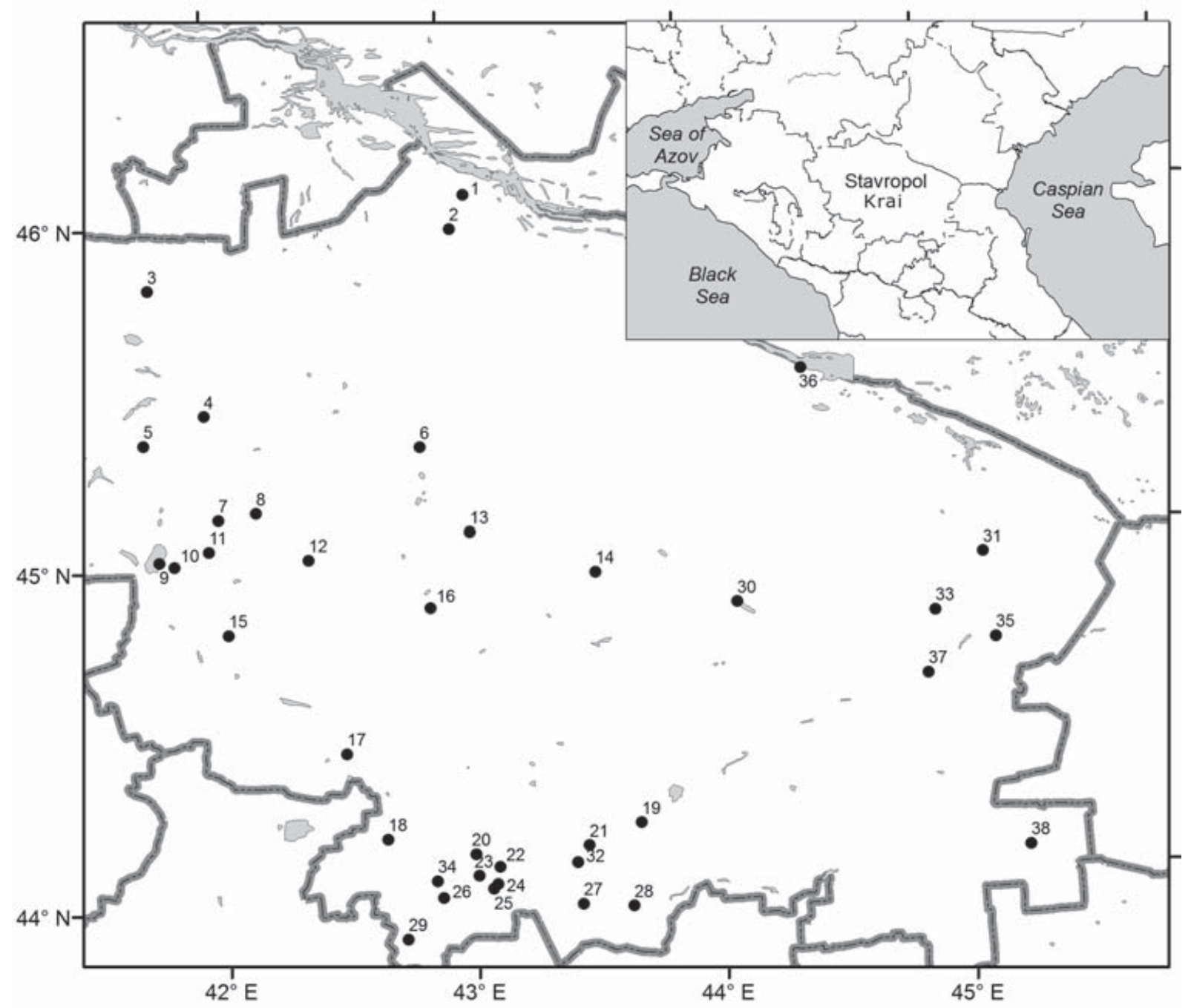

Map 1. Collecting localities and literature-based recprds in Stavropol Province.

Карта 1. Точки сбора и литературных указаний в Ставропольском крае.

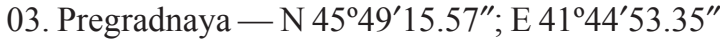

04. Donskoe - N 45 $26^{\prime} 59.45^{\prime \prime}$; E $41^{\circ} 58^{\prime} 0.35^{\prime \prime}$

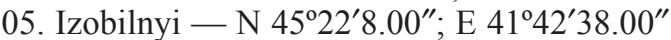

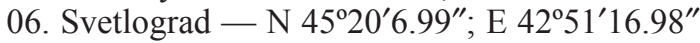

07. Mikhailovsk - N 45 $8^{\prime} 41.94^{\prime \prime}$; E $42^{\circ} 0^{\prime} 33.92^{\prime \prime}$

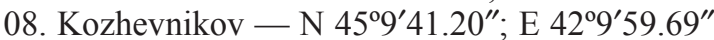

09. Sengileevskoe Reservoir - N 45 ${ }^{\circ} 1^{\prime} 39.52^{\prime \prime}$; E $41^{\circ} 45^{\prime} 44.27^{\prime \prime}$

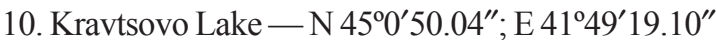

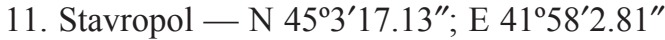

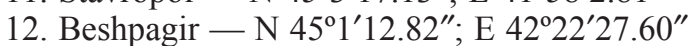

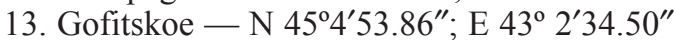

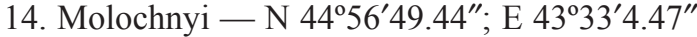

15. Strizhament Mt. - N 44 $48^{\prime} 33.15^{\prime \prime}$; E $42^{\circ} 2^{\prime} 6.58^{\prime \prime}$

16. Medvedskaya Mt. - N 44 $51^{\prime} 58.00^{\prime \prime}$; E $42^{\circ} 52^{\prime} 4.00^{\prime \prime}$

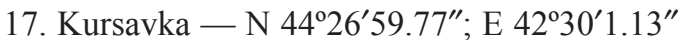

18. Suvorovskaya - N 44 $11^{\prime} 40.41^{\prime \prime}$; E $42^{\circ} 39^{\prime} 10.02^{\prime \prime}$

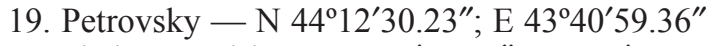

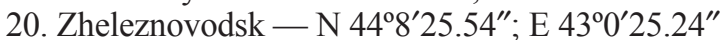

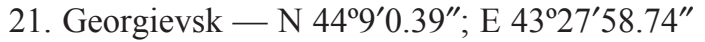

22. Inozemtsevo - $\mathrm{N} 44^{\circ} 6^{\prime} 0.00^{\prime \prime} ; \mathrm{E} 43^{\circ} 6^{\prime} 0.00^{\prime \prime}$

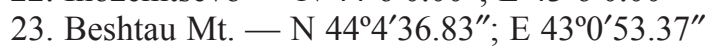

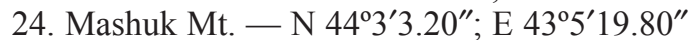

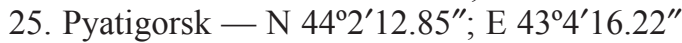

26. Essentuki (Essentukskaya) — N 44 ${ }^{\circ} 1^{\prime} 0.21^{\prime \prime}$; E $42^{\circ} 52^{\prime} 0.28^{\prime \prime}$

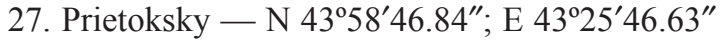

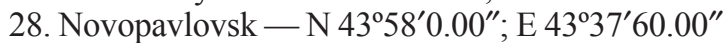

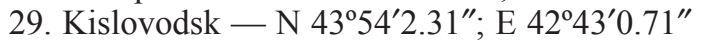

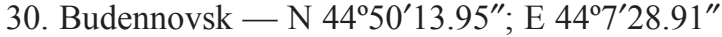

31. Velichaevskoe - N 44 $56^{\prime} 9.24^{\prime \prime}$; E $45^{\circ} 8^{\prime} 38.70^{\prime \prime}$

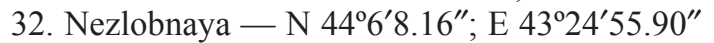

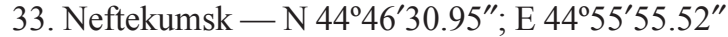

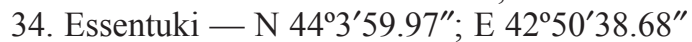

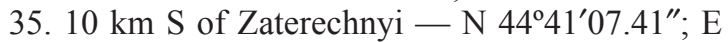
$45^{\circ} 10^{\prime} 15.13^{\prime \prime}$ 
36. $22 \mathrm{~km} \mathrm{NE}$ of Arzgir, Chograi Reservoir - N $45^{\circ} 30^{\prime} 20.20^{\prime \prime}$; E 44 $26^{\prime} 56.14^{\prime \prime}$ $08.86^{\prime \prime}$

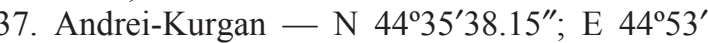

38. Sovkhoznyi - N 44 $04^{\prime} 23.36^{\prime \prime}$; E $45^{\circ} 15^{\prime} 02.61^{\prime \prime}$

\section{Annotated checklist of species}

\section{AGELENIDAE}

Agelena labyrinthica (Clerck, 1758)

MATERIAL. $1 \sigma^{7}$, Zheleznovodsk Distr., Inozemtsevo Vil., 25.06.2010 (P.I.); 5 O $^{\top} \sigma^{2}, 1$ + , Izobilnyi, 9.07-8.08.2011 (V.K.); 1 +, s.1., afforestation of Robinia pseudacacia, 18.07-21.08.2013 (V.K.); 2 O $^{\top} \sigma^{7}$, s.1., $5.07-15.08 .2014$ (V.K.).

Allagelena gracilens (C.L. Koch, 1841)

MATERIAL. 1 \%, Zheleznovodsk Distr., Inozemtsevo Vil., 8.09.2013 (P.I.).

Eratigena agrestis (Walckenaer, 1802)

RECORDS. Beshpagir [Logunov, Penney, 2004].

MATERIAL. 1 , , Stavropol, village community, inside house, 18.08.2000 (T.Kh.); 1 ㅇ, Zheleznovodsk Distr., Beshtau Mt., 8.09.2013 (P.I.); 3 O $^{7} \sigma^{7}, 4$ 우, Zheleznovodsk Distr., Inosemtsevo Vil., 8-9.09.2013 (P.I.); 2 Ơ $^{7}, 2$ 우, Izobilnyi, afforestation of Robinia pseudacacia, 18.07-21.08.2013 (V.K.); 1 o', s.l., small holding, 18.07-20.08.2013 (V.K.); 5 o $^{7} \sigma^{7}$, s.1., 5.07-15.08.2014 (V.K.). 2008

Tegenaria chumachenkoi Kovblyuk et Ponomarev,

RECORDS. Stavropol [Kovblyuk, Ponomarev, 2008].

Tegenaria domestica (Clerck, 1758)

MATERIAL. 1 +, Zheleznovodsk Distr., Inosemtsevo Vil., 8 9.09.2013 (P.I.).

Tegenaria lapicidinarum Spassky, 1934

MATERIAL. 3 OP, $22 \mathrm{~km} \mathrm{NE}$ of Arzgir Vil., reeds along Chograi Reservoir bank, 14-25.07.1987 (S.A.).

Tegenaria sp. 1

MATERIAL. $1 \overbrace{}^{7}, 20 \mathrm{~km} \mathrm{~S}$ of Stavropol, Strizhament Mt., 18.07.2013 (E.T.).

COMMENTS. This species is close to Tegenaria lehtineni (Guseinov, Marusik et Koponen, 2005) but differs from it in the shape of the palpal tibial apophysis and relatively long palpal patella. This species seems to be new to science.

Tegenaria sp. 2

RECORDS. Zheleznovodsk [Ponomarev, Mikhailov, 2007: Tegeneria pagana, misidentification].

MATERIAL. 2 o+, Zheleznovodsk Distr., Beshtau Mt., 26.06.2010 (P.I.); 1 q, same locality, 8.09.2013 (P.I.).

Pireneitega ovtchinnikovi Kovblyuk, Kastrygina, Marusik et Ponomarev, 2013

MATERIAL. 1 ㅇ, Pyatigorsk, S slope of Mashuk Mt., $600 \mathrm{~m}$ a.s.1., wood edge, 27.07.2008 (A.Z.).

\section{ARANEIDAE}

Aculepeira armida (Savigny et Audouin, 1826)

MATERIAL. $2 \sigma^{7} \sigma^{7}$, Apanasenkovskoe Distr., Manychskoe Vil., Manych-Gudilo Lake shore, 24.05.2005 (T.Kh.); $20^{7} \sigma^{7}$, s.l., steppe, 26-31.05.2005 (T.KH.); 1 \%, s.1., wheat field, 2.06.2005 (T.Kh.).

Araneus angulatus Clerck, 1758

MATERIAL. 1 ㅇ, Izobilnyi, 5.07-15.08.2014 (V.K.).

Araneus diadematus Clerck, 1758

MATERIAL. $1 \sigma^{7}, 1$, Pyatigorsk, S piedmont of Beshtau Mt., forest-steppe, 29.08.2008 (A.Z.); 1 \%, Essentuki, Essentukskaya Vil., 08.2003 (Yu.M.).

Argiope bruennichi (Scopoli, 1772)

RECORDS. Stavropol [Lukianov, 1897]; Beshpagir [Logunov, Penney, 2004].

MATERIAL. $1 \sigma^{7}, 1$, , Neftekumsk Distr., nr. Andrei-Kurgan Vil., 1-10.07.1987 (S.A.); 1 \&, Pyatigorsk, S piedmont of Beshtau Mt., forest-steppe, 29.08.2008 (A.Z.).

Argiope lobata (Pallas, 1772)

RECORDS. Beshpagir [Logunov, Penney, 2004].

Cercidia prominens (Westring, 1851)

MATERIAL. 1 q subad., Izobilnyi, 9.07-8.08.2011 (V.K.); 1 $\mathrm{O}^{7}$, s.1., afforestation of Robinia pseudacacia, 18.07-21.08.2013 (V.K.).

Gibbaranea bituberculata (Walckenaer, 1802)

MATERIAL. 1 +, Apanasenkovskoe Distr., Manychskoe Vil., afforestation, 26.05.2005 (T.Kh.).

Gibbaranea ullrichi (Hahn, 1835)

RECORDS. Manychskoe [Ponomarev et al., 2006].

MATERIAL. 2 우, Apanasenkovskoe Distr., Manychskoe Vil., steppe, 31.05.2005 (T.Kh.)

Hypsosinga albovittata (Westring, 1851)

RECORDS. Manychskoe [Ponomarev et al., 2006].

MATERIAL. 1 \%, Apanasenkovskoe Distr., Manychskoe Vil., steppe, 26.05.2005 (T.Kh.).

Hypsosinga pygmaea (Sundevall, 1831)

MATERIAL. $2 \mathrm{O}^{7} \mathrm{O}^{7}, 9$ 우, Apanasenkovskoe Distr., Manychskoe Vil., Manych-Gudilo Lake shore, 24-31.05.2005 (T.Kh.); $10^{7}$, Neftekumsk, saline floodplain meadow, 23-27.04.2012 (S.A.).

Larinioides folium (Schrank, 1803)

RECORDS. Petrovsky District [Titova, Egorova, 1978: Araneus cornutus].

MATERIAL. $74 \sigma^{7} \sigma^{7}, 40$ 우, Apanasenkovskoe Distr., Manychskoe Vil., Manych-Gudilo Lake shore, 24-31.05.2005 (T.Kh.); $1 \sigma^{7}$, s.l., saline meadow, 29.05.2005 (T.Kh.); 2 O $^{\top} \sigma^{7}, 1$, s.1., 19-30.05.2005 (S.P.).

Larinioides ixobolus (Thorell, 1873)

RECORDS. Petrovsky District [Titova, Egorova, 1978: Araneus].

Mangora acalypha (Walckenaer, 1802)

RECORDS. Beshpagir [Logunov, Penney, 2004]. 
MATERIAL. 1 ㅇ, Trunovskoe Distr., Donskoe Vil., steppe, 22.09.2000 (T.Kh.); 2 우, Apanasenkovskoe Distr., Manychskoe Vil., Manych-Gudilo Lake shore, 24.05.2005 (T.Kh.); $5 \sigma^{\top} \sigma^{\top}, 33$ +o, s.1., afforestation , 25-26.05.2005 (T.Kh.); 1 +, s.1., 1930.05.2005 (S.P.)

Neoscona adianta (Walckenaer, 1802)

RECORDS. Beshpagir [Logunov, Penney, 2004]; Zheleznovodsk [Ponomarev, Mikhailov, 2007].

MATERIAL. $1 \sigma^{7}, 20 \mathrm{~km}$ NE of Stavropol, Kozhevnikov Vil., steppe, 19.07.2000 (T.Kh.); 2 우, Sengileevskoe Reservoir, forb steppe, 17.09.2000 (T.Kh.); $1 \sigma^{\text {T }, ~} 1$ 9, Trunovskoe Distr., Donskoe Vil., steppe, 22.09.2000 (T.Kh.); $14 \sigma^{\top} \sigma^{7}, 13$ 우, Apanasenkovskoe Distr., Manychskoe Vil., Manych-Gudilo Lake shore, 24 31.05.2005 (T.Kh.); $67 \sigma^{7} \sigma^{7}, 33$ 우, s.1., steppe, 26-31.05.2005 (T.Kh.); 4 O $^{7}, 1$ ㅇ, s.1., saline meadow , 29.05.2005 (T.Kh.); 1 을 s.1., 19-30.05.2005 (S.P.); $2 \sigma^{7} \sigma^{7}$, Petrovsky Distr., Gofitskoe Vil., 18-29.06.2005 (E.Kh.); 1 б', Petrovsky Distr., Medvedskaya Mt. steppe, 19.06.2005 (E.Kh.); 1 ऽ', Zheleznovodsk Distr., Beshtau Mt., 28-30.07.2005 (A.R.).

Singa nitidula C.L. Koch, 1844

MATERIAL. 7 O $0^{7}$, Apanasenkovskoe Distr., Manychskoe Vil., Manych-Gudilo Lake shore, 26.05.2005 (T.Kh.).

\section{ATYPIDAE}

Atypus muralis Bertkau, 1890

RECORDS. Stavropol Province [Lukianov, 1897: Atypus anachoreta; Zonshtein, 1985].

\section{CLUBIONIDAE}

Clubiona brevipes Blackwall, 1841

RECORDS. Suvorovskaya [Mikhailov, 1992].

MATERIAL. 1 , Georgievsky Distr., Prietoksky Vil., 5.06.2003 (T.Kh.).

Clubiona caerulescens L. Koch, 1867

RECORDS. Pyatigorsk [Mikhailov, 1990].

Clubiona frisia Wunderlich et Schuett, 1995

RECORDS. Novopavlovsk [Mikhailov, 1990: Clubiona similis; 2003].

Clubiona golovatchi Mikhailov, 1990

RECORDS. Zheleznovodsk [Mikhailov, 1990, 2003].

MATERIAL. 1 \%, Izobilnyi, 9.07-8.08.2011 (V.K.).

Clubiona lutescens Westring, 1851

RECORDS. Pyatigorsk [Mikhailov, 1990].

Clubiona neglecta O. Pickard-Cambridge, 1862

RECORDS. Petrovsk [Mikhailov, 1990].

Clubiona pallidula (Clerck, 1758)

RECORDS. Suvorovskaya [Mikhailov, 1990].

Clubiona pseudoneglecta Wunderlich, 1994

MATERIAL. 2 우, Izobilnyi, grassy meadow, 18.0718.08.2013 (V.K.); 1 +, s.l., afforestation of Robinia pseudaca-

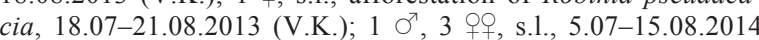
(V.K.).

\section{DICTYNIDAE} 1872)

Archaeodictyna consecuta (O. Pickard-Cambridge,

MATERIAL. $1 \sigma^{7}$, Neftekumsk Distr., $10 \mathrm{~km} \mathrm{~S}$ of Zaterechnyi Vil., sagebrush-grass steppe, 23-27.04.2012 (S.A.).

Argenna patula (Simon, 1874)

MATERIAL. $1 \sigma^{7}$, Neftekumsk, saline floodplain meadow, 23-27.04.2012 (S.A.).

Argenna subnigra (O. Pickard-Cambridge, 1861)

MATERIAL. 1 +, Apanasenkovskoe Distr., Manychskoe Vil., Manych-Gudilo Lake shore, 26.05.2005 (T.Kh.); $1 \mathrm{O}^{\text {', }}$, Neftekumsk, salt-pan with tamariks (Tamarix sp.), 23-27.04.2012 (S.A.).

Dictyna uncinata Thorell, 1856

MATERIAL. 1 , Stavropol, on chestnut (Aesculus hippocastanum), 30.04.2000 (T.Kh.); $1 \mathrm{O}^{7}$, s.1., Taman forest, 30.04.2000 (T.Kh.); 1 \%, Stavropol, village community, on rasberry (Rubus idaeus), 4.06.2000 (T.Kh.).

\section{DYSDERIDAE}

Dysdera crocata C.L. Koch, 1838

MATERIAL. $10^{7}$, Neftekumsk Distr., nr. Andrei-Kurgan Vil., 1-10.07.1987 (S.A.); $6 \mathrm{O}^{7} \sigma^{7}, 5$ 우, $22 \mathrm{~km} \mathrm{NE}$ of Arzgir Vil., reeds along Chograi Reservoir bank, 14-25.07.1987 (S.A.); 211 O $^{7} \sigma^{7}$,

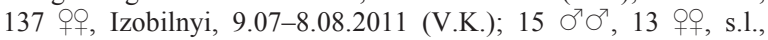
afforestation of Robinia pseudacacia, 18.07-21.08.2013 (V.K.); 21 Oొ $^{\top}, 9$ 우, s.1., 5.07-15.08.2014 (V.K.)

Dysdera dunini Deeleman-Reinhold, 1988

RECORDS. Zheleznovodsk [Dunin, 1992].

Harpactea rubicunda (C.L. Koch, 1838) 1992].

RECORDS. Zheleznovodsk, Stavropol [Dunin,

\section{ERESIDAE}

Eresus kollari Rossi, 1846

RECORDS. Manychskoe [Ponomarev et al., 2006: Eresus cinnaberinus].

MATERIAL. $1 \sigma^{7}$, Apanasenkovskoe Distr., Manychskoe Vil., afforestation, 25.05.2005 (T.Kh.); $2 \bigcirc^{7} \sigma^{7}$, Neftekumsk Distr., 10 $\mathrm{km} \mathrm{S}$ of Zaterechnyi Vil., foot of dune, 23-27.04.2012 (S.A.).

\section{EUTICHURIDAE}

Cheiracanthium erraticum (Walckenaer, 1802)

MATERIAL. $1 \sigma^{7}$, Apanasenkovskoe Distr., Manychskoe Vil., steppe, 31.05.2005 (T.Kh.); $2 O^{7} \sigma^{7}$, Petrovsky Distr., Gofitskoe Vil. , steppe, 18.06.2005 (E.Kh.)

Cheiracanthium gratum Kulczyński, 1897

MATERIAL. $1 \mathrm{O}^{2}$, Neftekumsk Distr., $10 \mathrm{~km} \mathrm{~S}$ of Zaterechnyi Vil., dune foothill, 23-27.04.2012 (S.A.).

Cheiracanthium mildei L. Koch, 1864

MATERIAL. 1 + , Izobilnyi, 9.07-8.08.2011 (V.K.). 
Cheiracanthium pelasgicum (C.L. Koch, 1837)

MATERIAL. 1 +, Neftekumsk Distr., nr. Andrei-Kurgan Vil., $1-10.07 .1987$ (S.A.). 1873

Cheiracanthium pennyi O. Pickard-Cambridge,

MATERIAL. $1 \bigcirc^{7}$, Sengileevskoe Reservoir, steppe, 11.06.2000 (T.Kh.); $5 \sigma^{\top} \sigma^{\top}, 2$ 우, Apanasenkovskoe Distr., Manychskoe Vil.,

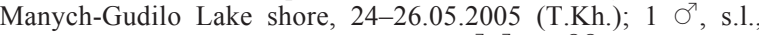
afforestation, 25.05.2005 (T.Kh.); $7 \sigma^{\top} \sigma^{7}, 3$ 우, s.l., steppe, 26.05.2005 (T.Kh.)

Cheiracanthium punctorium (Villers, 1789) V.K.).

MATERIAL. $1 \mathrm{O}^{7}$, Izobilnyi, small holding, 18.07-20.08.2013

Cheiracanthium virescens (Sundevall, 1832)

MATERIAL. 1 9, Izobilnyi, 5.07-15.08.2014 (V.K.).

\section{GNAPHOSIDAE} 1872)

Aphantaulax trifasciata (O. Pickard-Cambridge,

MATERIAL. $1 \mathrm{O}^{\top}$, Izobilnyi, 9.07-8.08.2011 (V.K.).

Berlandina cinerea (Menge, 1872)

RECORDS. Beshpagir [Logunov, Penney, 2004].

MATERIAL. 9 O $^{\top} \mathrm{O}^{7}, 1$ \% , Neftekumsk Distr., $10 \mathrm{~km} \mathrm{~S}$ of Zaterechnyi Vil., sagebrush-grass steppe, 23-27.04.2012 (S.A.); 8 $\mathrm{O}^{\top} \mathrm{O}^{\top}$, s.l., dune foothill, 23-27.04.2012 (S.A.); $14 \mathrm{O}^{7} \mathrm{O}^{7}$, Neftekumsk, salt-pan with tamarix, 23-27.04.2012 (S.A.).

Civizelotes caucasius (L. Koch, 1866)

MATERIAL. 5 Or $^{7}$, 2 + + , Izobilnyi, 9.07-8.08.2011 (V.K.).

Civizelotes gracilis (Canestrini, 1868)

MATERIAL. 2 O $^{7}$, 1 ㅇ, Izobilnyi, 9.07-8.08.2011 (V.K.); 1 +, s.1., 5.07-15.08.2014 (V.K.).

Drassodes lapidosus (Walckenaer, 1802)

RECORDS. Beshpagir [Logunov, Penney, 2004].

MATERIAL. $20^{7} \sigma^{7}$, Apanasenkovskoe Distr., Manychskoe Vil., 25-30.05.2005 (T.Kh.); 1 O$^{7}, 1$ + , Izobilnyi, 9.07-8.08.2011 (V.K.); 2 ofo, s.l., afforestation of Robinia pseudacacia, 18.0721.08.2013 (V.K.); 1 + , s.1., 5.07-15.08.2014 (V.K.).

Drassodes pubescens (Thorell, 1856)

MATERIAL. 2 90, Izobilnyi, 9.07-8.08.2011 (V.K.); 1 ㅇ, s.1., grassy meadow, 18.07-18.08.2013 (V.K.); 5 우, s.l., afforestation of Robinia pseudacacia, 18.07-21.08.2013 (V.K.); 5 +o, s.1., 5.0715.08.2014 (V.K.)

Drassyllus praeficus (L. Koch, 1866)

MATERIAL $10^{7}$, Apanasenkovskoe Distr., Manychskoe Vil., Manych-Gudilo Lake shore, 24.05.2005 (T.Kh.); 18 우, Izobilnyi, 9.07-8.08.2011 (V.K.); 1 ㅇ, s.1., grassy meadow, 18.07-18.08.2013 (V.K.); 2 of , s.l., afforestation of Robinia pseudacacia, 18.0721.08.2013 (V.K.); 2 우, s.1., 5.07-15.08.2014 (V.K.).

Drassyllus pusillus (C.L. Koch, 1833)

MATERIAL. $1 \sigma^{7}$, Stavropol, Chlinskiy forest, 7.05.2000 (T.Kh.); $3 \bigcirc^{\top} \sigma^{\top}, 1$ q, Apanasenkovskoe Distr., Manychskoe Vil., windbreak, 29.05-2.06.2005 (T.Kh.); $2 \sigma^{7} \sigma^{7}$, s.1., 25.05-3.06.2005 (T.Kh.); $2 \bigcirc^{7} \sigma^{7}, 13$ 우, Izobilnyi, 9.07-8.08.2011 (V.K.); 1 + , s.l., afforestation of Robinia pseudacacia, 18.07-21.08.2013, (V.K.); 2
우, s.1., 5.07-15.08.2014 (V.K.); 1 O , Neftekumsk, salt-pan with tamarix, 23-27.04.2012 (S.A.).

Drassyllus shaanxiensis Platnick et Song, 1986

Fig. 1 .

MATERIAL. $3 \sigma^{7} \sigma^{7}$, Neftekumsk, saline floodplain meadow, 23-27.04.2012 (S.A.).

COMMENTS. This species was originally described from eastern China [Platnick, Song, 1986]. Recently, some females of this species were found in Tyuleniy island in the north-western part of Caspian Sea [Ponomarev et al., 2011]. The illusration of male palp of the studied specimens is given in Fig. 1.

Drassyllus vinealis (Kulczyński in Chyser et Kulczyński, 1897)

MATERIAL. $1 \sigma^{7}$, Apanasenkovskoe Distr., Manychskoe Vil., 25-30.05.2005 (T.Kh.); 1 o , s.1., afforestation, 29.05-2.06.2005 (T.Kh.); 12 우 Izobilnyi, 9.07-8.08.2011 (V.K.); 2 + + , s.1., 5.0715.08.2014 (V.K.)

Gnaphosa cumensis Ponomarev, 1981

MATERIAL. $27 \sigma^{7} \sigma^{7}, 17$ 우, Neftekumsk Distr., nr. AndreiKurgan Vil., 1-10.07.1987 (S.A.); $1 \sigma^{7}, 8$ 우, 22 km NE of Arzgir Vil., reeds along Chograi Reservoir bank, 14-25.07.1987 (S.A.).

Gnaphosa dolosa O. Herman, 1879

MATERIAL. 1 \% , Chograi Reservoir bank, 23.06 .2000 (T.Kh.); $1 \mathrm{O}^{\top}$, Apanasenkovskoe Distr., Manychskoe Vil., Manych-Gudilo Lake shore, 31.05.2005 (T.Kh.); 1 \%, Neftekumsk, saline floodplain meadow, 23-27.04.2012 (S.A.); $1 \mathrm{\sigma}^{7}$, Izobilnyi, 5.0715.08.2014 (V.K.).

Gnaphosa leporina (L. Koch, 1866)

MATERIAL. $232 \sigma^{7} \sigma^{7}, 70$ 오, Izobilnyi, 9.07-8.08.2011

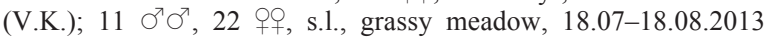
(V.K.); $100^{7} 0^{7}, 5$ 우, s.l., afforestation of Robinia pseudacacia, 18.07-21.08.2013 (V.K.); 6 O $^{7} \sigma^{7}, 7$ ㅇ, s.1., 5.07-15.08.2014 (V.K.); $20^{7} 0^{7}$, Neftekumsk Distr., $10 \mathrm{~km} \mathrm{~S}$ of Zaterechnyi Vil., sagebrushgrass steppe, 23-27.04.2012 (S.A.); 13 우오, Neftekumsk, salt-pan with tamarix, 23-27.04.2012 (S.A.).

Gnaphosa lucifuga (Walckenaer, 1802) 1978].

RECORDS. Petrovsky District [Titova, Egorova,

Gnaphosa mongolica Simon1895

RECORDS. Beshpagir [Logunov, Penney, 2004].

MATERIAL. $80^{7} \sigma^{7}, 200$, Neftekumsk Distr., $10 \mathrm{~km} \mathrm{~S}$ of Zaterechnyi Vil., dune foothill, 23-27.04.2012 (S.A.). 1992

Gnaphosa saurica Ovtsharenko, Platnick et Song,

MATERIAL. $3 \sigma^{7} \sigma^{7}, 2$ 우, Neftekumsk Distr., nr. Andrei-Kur-

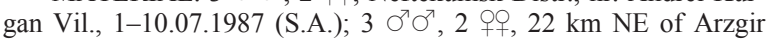
Vil., reeds along Chograi Reservoir bank, 14-25.07.1987 (S.A.).

Gnaphosa taurica Thorell, 1875

MATERIAL. 1 O$^{7}$, Stavropol, Budarka Mt., 9.03.2001 (T.Kh.); $2 \sigma^{7} \sigma^{7}$, Apanasenkovskoe Distr., Manychskoe Vil., Manych-Gudilo Lake shore, 24.05.2005 (T.Kh.).

Haplodrassus bohemicus Miller et Buchar, 1977

RECORDS. Manychskoe [Ponomarev, Tsvetkov, 2006]. 

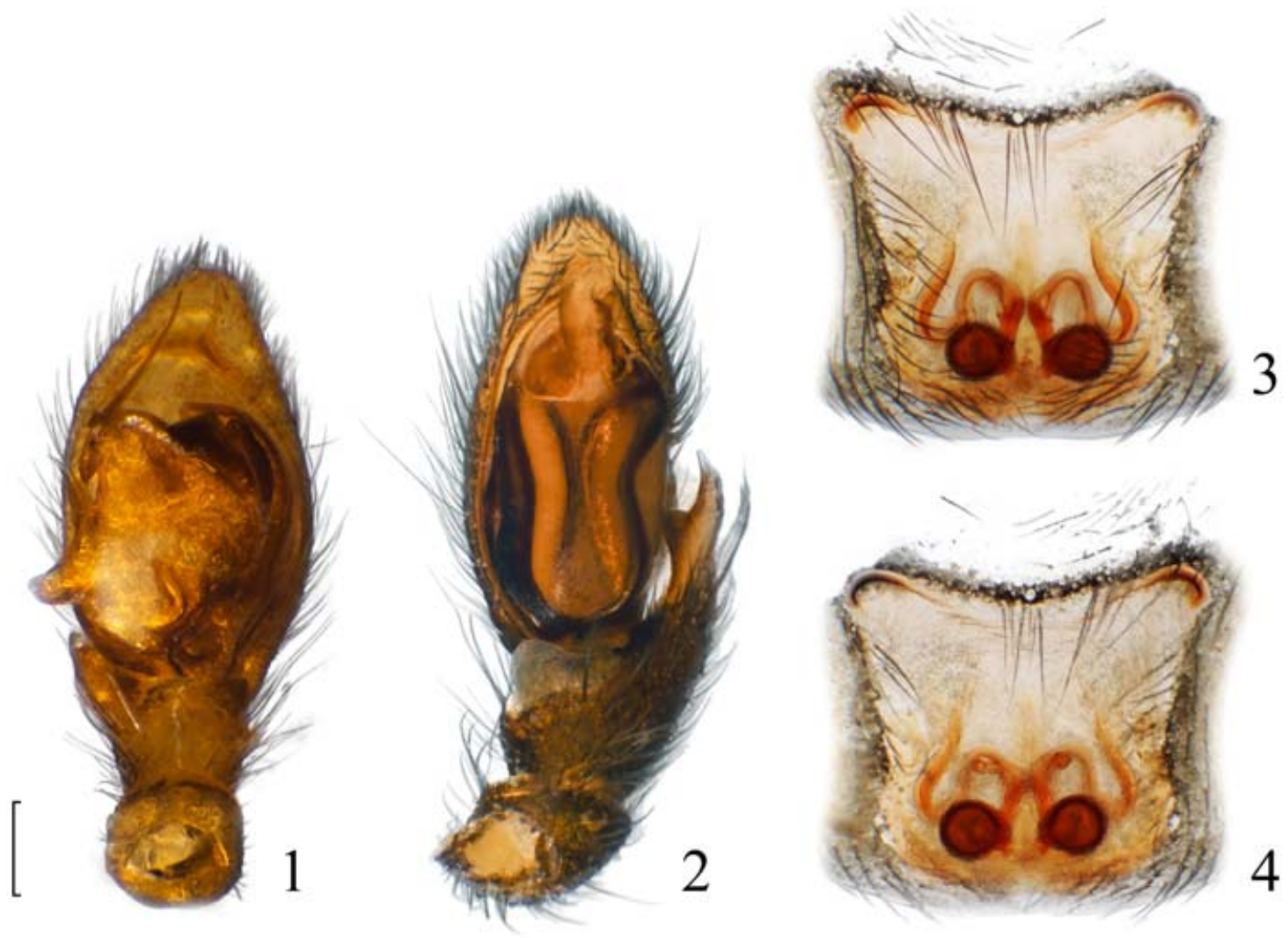

Figs. 1-4. Copulatory organs of Drassyllus shaanxiensis (1), Poecilochroa senilis (2) and Zelotes engenei (3, 4): 1, 2 - male palp, ventral view; 3 - epigyne, ventral view; 4 - epigyne, dorsal view. Scale bars: $0.2 \mathrm{~mm}$.

Рис. 1-4. Копулятивные органы Drassyllus shaanxiensis (1), Poecilochroa senilis (2) и Zelotes engenei (3, 4): 1, 2 — пальпа самца, вид снизу; 3 - эпигина, вид снизу; 4 - эпигина, вид сверху. Масштаб 0,2 мм.

MATERIAL. 4 우, Izobilnyi, 9.07-8.08.2011 (V.K.).

Haplodrassus dalmatensis (L. Koch, 1866)

MATERIAL. $20^{7} 0^{7}$, Apanasenkovskoe Distr., Manychskoe Vil., 25-30.05.2005 (T.Kh.); 1 + , s.1., afforestation, 29.05-2.06.2005 (T.Kh.).

Haplodrassus minor (O. Pickard-Cambridge, 1879) MATERIAL. 2 O' $^{7}$, Izobilnyi, 9.07-8.08.2011 (V.K.).

Haplodrassus signifer (C.L. Koch, 1839) 1978].

RECORDS. Petrovsky District [Titova, Egorova,

MATERIAL. $1 \sigma^{7}$, Apanasenkovskoe Distr., Manychskoe Vil., 19-20.05.2005 (S.P.); $10^{7}$, Neftekumsk Distr., $10 \mathrm{~km} \mathrm{~S}$ of Zaterechnyi Vil., sagebrush-grass steppe, 23-27.04.2012 (S.A.).

Micaria albovittata (Lucas, 1846)

MATERIAL. 6 오, Izobilnyi, 9.07-8.08.2011 (V.K.); 1 ㅇ, s.1., 5.07-15.08.2014 (V.K.).

Micaria dives (Lucas, 1846)

MATERIAL. 1 +, Izobilnyi, 9.07-8.08.2011 (V.K.).

Micaria formicaria (Sundevall, 1831)

MATERIAL. 4 O7 $\sigma^{7}$, Izobilnyi, 9.07-8.08.2011 (V.K.); 1 ㅇ, s.l., afforestation of Robinia pseudacacia, 18.07-21.08.2013 (V.K.).
Micaria pallipes (Lucas, 1846)

MATERIAL. $5 \sigma^{7} \sigma^{7}, 1$, Neftekumsk, salt-pan with tamarix, 23-27.04.2012 (S.A.).

Micaria pulicaria (Sundevall, 1831)

MATERIAL. 1 ㅇ, Izobilnyi, 9.07-8.08.2011 (V.K.); $1 \mathrm{O}^{7}$, Neftekumsk, saline floodplain meadow, 23-27.04.2012 (S.A.).

Micaria rossica Thorell, 1875

RECORDS. Petrovsky District [Titova, Egorova, 1978; Mikhailov, 1987].

MATERIAL. $10^{7}$, Apanasenkovskoe Distr., Manychskoe Vil., 25-30.05.2005 (T.Kh.); 1 ๙ $^{7}, 1$ ㅇ, s.1., afforestation, 29.052.06.2005 (T.Kh.); 1 ㅇ, Izobilnyi, 9.07-8.08.2011 (V.K.); 5 O $^{7} \sigma^{7}$, 1 + , Neftekumsk, saline floodplain meadow, 23-27.04.2012 (S.A.); $1 \sigma^{7}$, s.1., salt-pan with tamarix, 23-27.04.2012 (S.A.); $2 \sigma^{7} \sigma^{\prime \prime}$, Neftekumsk Distr., $10 \mathrm{~km} \mathrm{~S}$ of Zaterechnyi Vil., sagebrush-grass steppe, 23-27.04.2012 (S.A.); 1 Oै $^{2} 1$ \%, s.l., dune foothill, 2327.04.2012 (S.A.).

Nomisia aussereri (L. Koch, 1872)

RECORDS. Beshpagir [Logunov, Penney, 2004].

Phaeocedus braccatus (L. Koch, 1866)

MATERIAL. 1 +, Neftekumsk Distr., nr. Andrei-Kurgan Vil., 1-10.07.1987 (S.A.); 7 O$^{7} \mathrm{O}^{7}, 4$ 우, Izobilnyi, 9.07-8.08.2011 (V.K.); 9 우, s.1., afforestation of Robinia pseudacacia, 18.07-21.08.2013 (V.K.); 1 ○’, 15 우, s.1., 5.07-15.08.2014 (V.K.). 
Poecilochroa senilis (O. Pickard-Cambridge, 1872) Fig. 2.

MATERIAL. $1 \sigma^{7}$, Neftekumsk Distr., nr. Andrei-Kurgan Vil., $1-10.07 .1987$ (S.A.).

COMMENTS. In Russia, to date, this rare species has only been found in the Crimea [Kovblyuk, Tuneva, 2009] and Tyuleniy and Chechen islands in Daghestan [Ponomarev et al., 2011].

Sosticus loricatus (L. Koch, 1866)

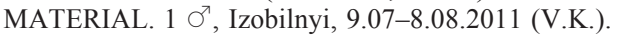

Talanites fagei Spassky,1938

RECORDS. Suvorovskaya [Platnick, Ovtsharenko, 1991]; Manychskoe [Ponomarev, 2008a].

Trachyzelotes adriaticus (Caporiacco, 1951)

MATERIAL. 1 + $22 \mathrm{~km}$ NE of Arzgir Vil., reeds along Chograi Reservoir bank, 14-25.07.1987 (S.A.); $12 \bigcirc^{7} \sigma^{7}, 7$ 우, Neftekumsk Distr., nr. Andrei-Kurgan Vil., 1-10.07.1987 (S.A.).

Trachyzelotes cumensis (Ponomarev, 1979)

MATERIAL. 2 오, Neftekumsk Distr., nr. Andrei-Kurgan Vil., 1-10.07.1987 (S.A.); 1 \%, $22 \mathrm{~km} \mathrm{NE}$ of Arzgir Vil., reeds along Chograi Reservoir bank, 14-25.07.1987 (S.A.); 3 O $^{7}$ O $^{7}$, Neftekumsk, salt-pan with tamarix, 23-27.04.2012 (S.A.).

Trachyzelotes malkini Platnick et Murphy, 1984

RECORDS. Petrovsky District [Titova, Egorova, 1978: Zelotes barbatus, misidentification].

MATERIAL. 1 +, Neftekumsk Distr., nr. Andrei-Kurgan Vil., 1-10.07.1987 (S.A.); 1 O$^{7}, 1$ ㅇ, $22 \mathrm{~km} \mathrm{NE}$ of Arzgir Vil., reeds along Chograi Reservoir bank, 14-25.07.1987 (S.A.); 1 Oै, Apanasenkovskoe Distr., Manychskoe Vil., 25-30.05.2005 (T.Kh.); 2 $\sigma^{7} \sigma^{7}, 21$ 우, Izobilnyi, 9.07-8.08.2011 (V.K.); $1 \sigma^{7}, 1$ ㅇ, s.l., afforestation of Robinia pseudacacia, 18.07-21.08.2013 (V.K.); 7 우오, s.1., $5.07-15.08 .2014$ (V.K.).

Trachyzelotes manytchensis Ponomarev et Tsvetkov, 2006

RECORDS. Izobilnyi [Ponomarev et al., 2016].

Trachyzelotes pedestris (C.L. Koch, 1837)

MATERIAL. 1 O?, 7 ㅇ, Izobilnyi, 9.07-8.08.2011 (V.K.); 3 ㅇ, s.l., afforestation of Robinia pseudacacia, 18.07-21.08.2013 (V.K.); 22 우, s.1., 5.07-15.08.2014 (V.K.).

Urozelotes rusticus (L. Koch, 1872)

MATERIAL. 1 ㅇ, Izobilnyi, 18.07-21.08.2013 (V.K.).

Zelotes azsheganovae Esyunin et Efimik, 1992

MATERIAL. $1 \mathrm{O}^{7}, 1$ ㅇ, Izobilnyi, 5.07-15.08.2014 (V.K.).

Zelotes electus (C.L. Koch, 1839)

RECORDS. Beshpagir [Logunov, Penney, 2004].

MATERIAL. $1 \sigma^{7}, 18$ + + , Izobilnyi, 9.07-8.08.2011 (V.K.); 1 $\mathrm{O}^{7}, 1$ ㅇ, s.1., afforestation of Robinia pseudacacia, 18.07-21.08.2013 (V.K.); 1 o , 5 웅, s.1., 5.07-15.08.2014 (V.K.); 1 ○ౌ, 2 우, Neftekumsk Distr., $10 \mathrm{~km} \mathrm{~S}$ of Zaterechnyi Vil., sagebrush-grass steppe, 23-27.04.2012 (S.A.); 5 O $^{\top} \sigma^{\top}, 5$ OQ , Neftekumsk, salt-pan with tamarix, 23-27.04.2012 (S.A.). 2009

Zelotes eugenei Kovblyuk in Kovblyuk et Tuneva,

Figs. 3, 4.
MATERIAL. 2 ㅇ, Neftekumsk Distr., nr. Andrei-Kurgan Vil., 1-10.07.1987 (S.A.); 4 +, $22 \mathrm{~km}$ NE of Arzgir Vil., reeds along Chograi Reservoir bank, 14-25.07.1987 (S.A.).

COMMENTS. This rare species was described from Kerch Peninsula of the Crimea [Kovblyuk, Tuneva, 2009] and was then recorded from the coast of Taganrog Bay [Ponomarev et al., 2016].

Zelotes fuscus (Thorell, 1875)

MATERIAL. $1 \sigma^{7}$, Apanasenkovskoe Distr., Manychskoe Vil., afforestation, 29.05-2.06.2005 (T.Kh.); $120 \mathrm{O}^{7} \sigma^{7}, 130$ 우, Izobilnyi, 9.07-8.08.2011 (V.K.); $104 \mathrm{O}^{7} \sigma^{7}, 92$ 우, s.l., afforestation of Robinia pseudacacia, 18.07-21.08.2013 (V.K.); $47 \sigma^{7} \sigma^{7}, 28$ 우, s.1., $5.07-15.08 .2014$ (V.K.). 1897)

Zelotes hermani (Chyser in Chyser et Kulczyński,

MATERIAL. 4 우, Izobilnyi, 9.07-8.08.2011 (V.K.); $10^{7}$, s.1., afforestation of Robinia pseudacacia, 18.07-21.08.2013 (V.K.).

Zelotes latreillei (Simon, 1878)

MATERIAL. 1 9, Izobilnyi, 9.07-8.08.2011 (V.K.); $50^{7} \sigma^{7}$, s.1., afforestation of Robinia pseudacacia, 18.07-21.08.2013 (V.K.); $3 \mathrm{O}^{7} 0^{7}, 4$ 우, s.1., $5.07-15.08 .2014$ (V.K.).

Zelotes longipes (L. Koch, 1866)

RECORDS. Kislovodsk [Spassky, 1936: Prosthesima serotina].

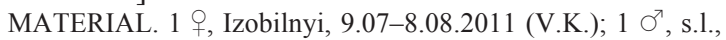
5.07-15.08.2014 (V.K.).

Zelotes mundus (Kulczyński in Chyzer et Kulczyński, 1897)

MATERIAL. $2 O^{7} \sigma^{7}$, Neftekumsk, saline floodplain meadow, 23-27.04.2012 (S.A.).

Zelotes prishutovae Ponomarev et Tsvetkov, 2006

MATERIAL. $1 \sigma^{7}$, Neftekumsk Distr., nr. Andrei-Kurgan Vil., $1-10.07 .1987$ (S.A.).

Zelotes puritanus Chamberlin, 1922

MATERIAL. $3 \sigma^{7} \sigma^{7}$, Neftekumsk Distr., $10 \mathrm{~km} \mathrm{~S}$ of Zaterechnyi Vil., dune foothill, 23-27.04.2012 (S.A.).

Zelotes tenuis (L. Koch, 1866)

Figs. 5, 6 .

MATERIAL. 2 O$^{7} 0^{7}$, Izobilnyi, 9.07-8.08.2011 (V.K.).

COMMENTS. In Russia, to date, this rare species has been found in the Crimea [Kovblyuk, 2005] and Krasnodar Province [Esyunin, Tuneva, 2012] only.

Zelotes sp.

Fig. 7.

MATERIAL. $10^{7}, 22 \mathrm{~km}$ NE of Arzgir Vil., reeds along Chograi Reservoir bank, 14-25.07.1987 (S.A.).

COMMENTS. The bulbus structure in this species resembles that of the puritanus group in the genus Zelotes. However, it differs from other species of the puritanus group in having the elongated and thin palpal tibial apophysis. The only collected male does not allow us to identify this species with confidence.

Gnaphosidae gen. sp.

Figs. 8, 9. 


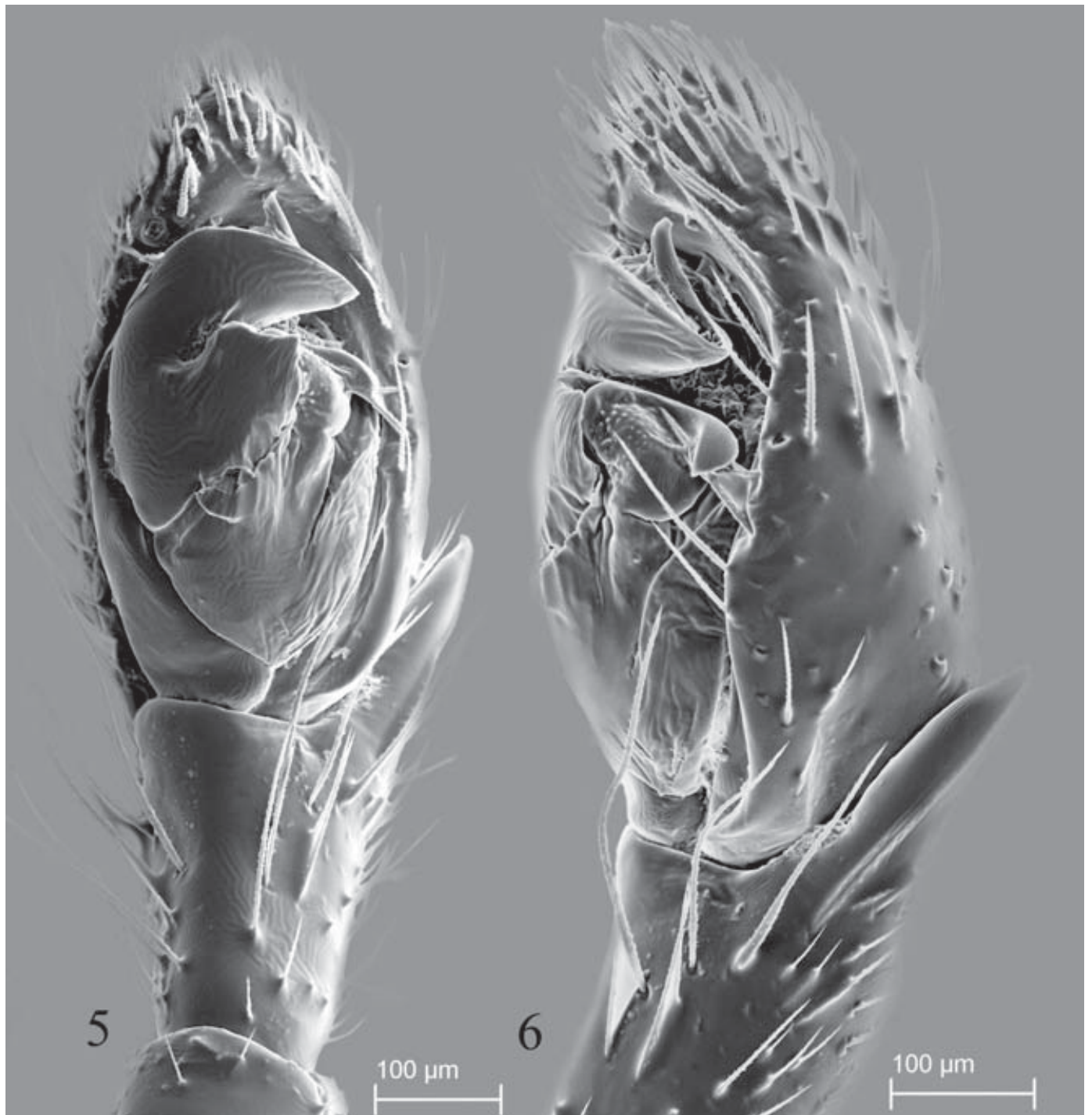

Figs. 5, 6. Male palp of Zelotes tenuis: 5 - ventral view; 6 - lateral view.

Рис. 5, 6. Пальпа самца Zelotes tenuis: 5 - вид снизу; 6 - вид сбоку.

MATERIAL. $1 \sigma^{7}, 22 \mathrm{~km}$ NE of Arzgir Vil., reeds along Chograi Reservoir bank, 14-25.07.1987 (S.A.).

COMMENTS. We have been unable to identify a genus name for this species.

\section{LINYPHIIDAE}

Acartauchenius scurrilis (O. Pickard-Cambridge, 1872)

MATERIAL. $1 \sigma^{\top}$, Neftekumsk Distr., $10 \mathrm{~km} \mathrm{~S}$ of Zaterechnyi Vil., dune foothill, 23-27.04.2012 (S.A.).

Agyneta rurestris (C.L. Koch, 1836)

MATERIAL. $1 \sigma^{7}$, Stavropol, inside an apartment house, 17.06.2000 (T.Kh.); 1 ㅇ, Apanasenkovskoe Distr., Manychskoe

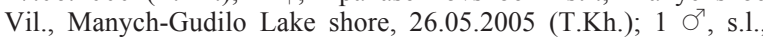
afforestation, 29.05-2.06.2005 (T.Kh.); 1 오․ Izobilnyi, 9.078.08.2011 (V.K.); 1 o $^{7} 1$ 1 , Neftekumsk, saline floodplain meadow, 23-27.04.2012 (S.A.).
Agyneta saaristoi Tanasevitch, 2000

MATERIAL. $1 \sigma^{\text {T}}$, Pyatigorsk, S slope of Mashuk Mt., $600 \mathrm{~m}$ a.s.1., forest edge, 27.07.2008 (A.Z.).

Centromerus minor Tanasevitch, 1990

RECORDS. Zheleznovodsk [Tanasevitch, 1990].

Dicymbium nigrum (Blackwall, 1834)

RECORDS. Pyatigorsk [Tanasevitch, 1987].

Diplocephalus picinus (Blackwall, 1841)

RECORDS. Pyatigorsk [Tanasevitch, 1987].

Diplostyla concolor (Wider, 1834)

RECORDS. Zheleznovodsk [Tanasevitch, 1987].

MATERIAL. 1 ㅇ, Izobilnyi, 9.07-8.08.2011 (V.K.); 1 ㅇ, Zheleznovodsk Distr., Beshtau Mt., 8.09.2013 (P.I.). 

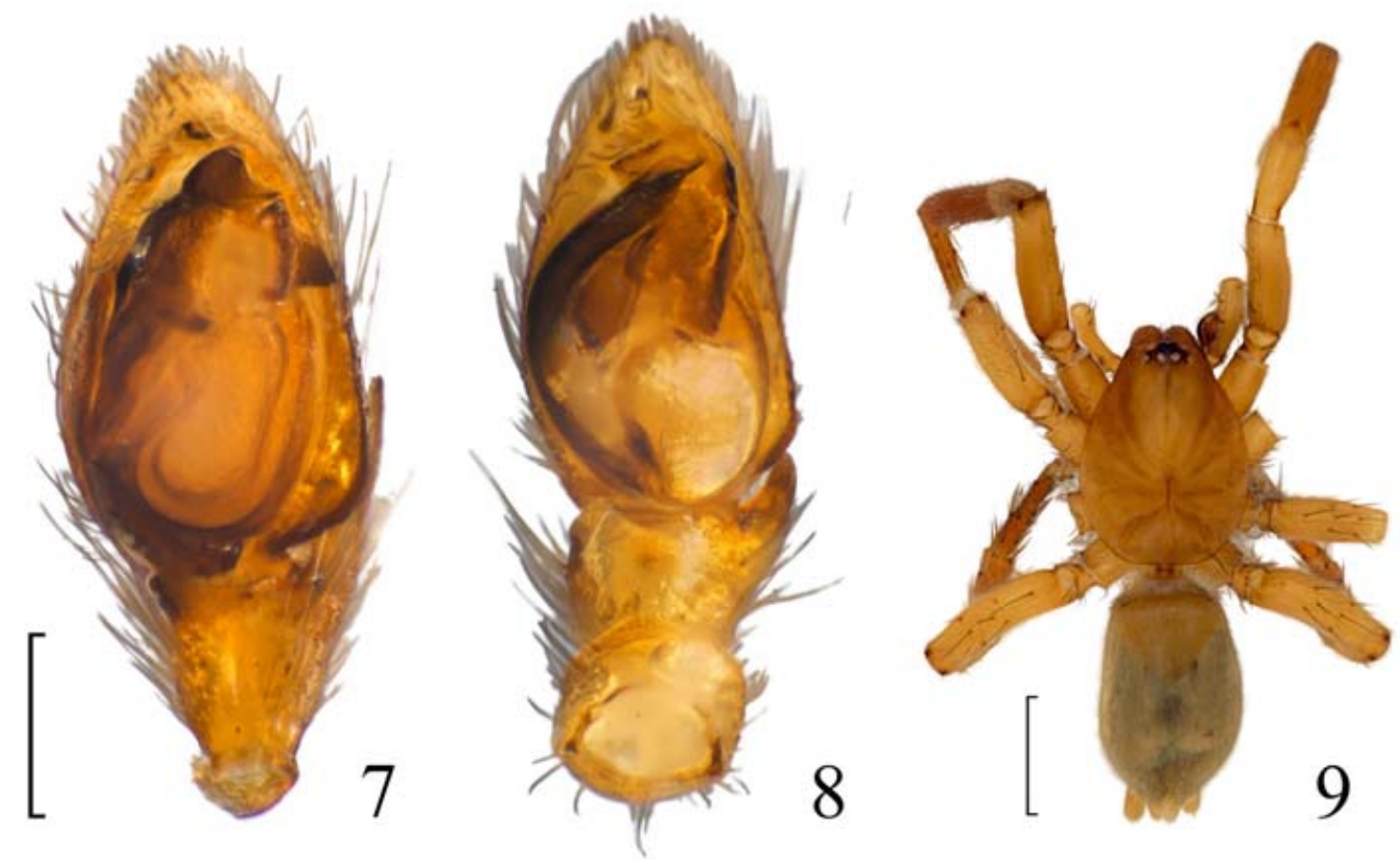

Figs. 7-9. Zelotes sp. (7) and Gnaphosidae gen. sp. (8, 9): 7, 8 - male palp, ventral view; 9 - male, dorsal view. Scale bars: figs. 7, $8-0.2 \mathrm{~mm}$; fig. $9-1 \mathrm{~mm}$.

Рис. 7-9. Zelotes sp. (7) и Gnaphosidae gen. sp. (8, 9): 7, 8 - пальпа самца, вид снизу; 9 - самец, вид сверху. Масштаб: рис. 7,8 - 0,2 мм; рис. $9-1$ мм.

Entelecara acuminata (Wider, 1834)

RECORDS. Zheleznovodsk [Tanasevitch, 1987].

Erigonoplus globipes (L. Koch, 1872)

MATERIAL. 1 +, Izobilnyi, 9.07-8.08.2011 (V.K.).

Erigonoplus spinifemuralis Dimitrov, 2003

MATERIAL. $10^{7}$, Neftekumsk Distr., nr. Andrei-Kurgan Vil., $1-10.07 .1987$ (S.A.).

Gnathonarium dentatum (Wider, 1834)

MATERIAL. 1 , , Neftekumsk, saline floodplain meadow, 2327.04.2012 (S.A.)

Incestophantes amotus (Tanasevitch, 1990)

RECORDS. Kislovodsk [Ponomarev et al., 2006; Ponomarev, Mikhailov, 2007].

Lepthyphantes leprosus (Ohlert, 1867)

RECORDS. Beshpagir [Logunov, Penney, 2004].

Linyphia triangularis (Clerck, 1758)

RECORDS. Beshpagir [Logunov, Penney, 2004].

Maso sundevalli (Westring, 1851)

RECORDS. Pyatigorsk [Tanasevitch, 1987].

Mecynargus minutipalpis Gnelitsa, 2011

MATERIAL. 1 , , Neftekumsk Distr., nr. Andrei-Kurgan Vil., 1-10.07.1987 (S.A.); 2 우, Neftekumsk, saline floodplain meadow, 23-27.04.2012 (S.A.). 1872)

Metopobactrus prominulus (O. Pickard-Cambridge, RECORDS. Kursavka, Georgievsk [Tanasevitch, 1987].

Micrargus subaequalis (Westring, 1851)

RECORDS. Zheleznovodsk [Tanasevitch, 1987].

Microneta viaria (Blackwall, 1841)

RECORDS. Zheleznovodsk , Pyatigorsk [Tanasevitch, 1987].

Microlinyphia pusilla (Sundevall, 1830)

MATERIAL. $20^{7} 0^{7}$, Apanasenkovskoe Distr., Manychskoe Vil., afforestation, 29.05-2.06.2005 (T.Kh.); 1 O , 4 우, s.l., Manych-Gudilo Lake shore, 31.05.2005 (T.Kh.).

Oedothorax apicatus (Blackwall, 1850)

MATERIAL. 1 +, Neftekumsk Distr., nr. Andrei-Kurgan Vil., 1-10.07.1987 (S.A.); 1 , Neftekumsk, saline floodplain meadow, 23-27.04.2012 (S.A.); 1 ค , Izobilnyi, 9.07-8.08.2011 (V.K.).

Sauron? sp.

MATERIAL. $1 \sigma^{\top}$, Neftekumsk, salt-pan with tamarix, 2327.04.2012 (S.A.).

Savignia frontata (Blackwall, 1833)

RECORDS. Pyatigorsk [Tanasevitch, 1987].

Silometopus reussi (Thorell, 1871)

MATERIAL. 2 io, Neftekumsk, saline floodplain meadow, 23-27.04.2012 (S.A.). 
Stemonyphantes lineatus (Linnaeus, 1758)

MATERIAL. $1 \sigma^{7}, 1$, Neftekumsk, salt-pan with tamarix, 23-27.04.2012 (S.A.).

Styloctetor compar (Westring, 1861)

MATERIAL. $1 \sigma^{7}$, Neftekumsk, saline floodplain meadow, 2327.04.2012 (S.A.), det. A.V. Tanasevitch.

Styloctetor romanus (O. Picard-Cambridge, 1872)

RECORDS. Beshpagir [Logunov, Penney, 2004].

MATERIAL. $1 \sigma^{7}$, Apanasenkovskoe Distr., Manychskoe Vil., afforestation, 26.05.2005 (T.Kh.).

Tenuiphantes mengei (Kulczyñski, 1887)

RECORDS. Zheleznovodsk, Pyatigorsk [Tanasevitch, 1987: Lepthyphantes].

MATERIAL. 2 우, Pyatigorsk, S slope of Mashuk Mt., $600 \mathrm{~m}$ a.s.1., forest edge, 27.07.2008 (A.Z.); $1 \overbrace{}^{\top}, 2$ 우, Zheleznovodsk Distr., Beshtau Mt., 26.06.2010 (P.I.); 2 우, s.1., 8.09.2013 (P.I.); 1 ㅇ, Zheleznovodsk Distr., Inozemtsevo Vil., 8-9.09.2013 (P.I.)

Tenuiphantes tenuis (Blackwall, 1852)

RECORDS. Beshpagir [Logunov, Penney, 2004].

Trichoncoides piscator (Simon, 1884)

MATERIAL. 2B\&, Neftekumsk Distr., nr. Andrei-Kurgan Vil., $1-10.07 .1987$ (S.A.)

Walckenaeria antica (Wider, 1834)

RECORDS. Zheleznovodsk [Tanasevitch, 1987].

MATERIAL. 1 +, Stavropol, Budarka Mt., 9.03.2000 (T.Kh.). 1878

Walckenaeria atrotibialis O. Pickard-Cambridge,

RECORDS. Pyatigorsk [Tanasevitch, 1987].

Walckenaeria cucullata (C.L. Koch, 1836)

RECORDS. Pregradnaya [Tanasevitch, 1990].

\section{LIOCRANIDAE}

Agroeca cuprea Menge, 1873

MATERIAL. 24 우, Izobilnyi, 9.07-8.08.2011 (V.K.); 4 우, s.l., 5.07-15.08.2014 (V.K.).

Agroeca lusatica (L. Koch, 1875)

MATERIAL. 7 우, Izobilnyi, 9.07-8.08.2011 (V.K.); 2 우, s.l., 5.07-15.08.2014 (V.K.).

Liocranoeca spasskyi Ponomarev, 2007

MATERIAL. $4 \mathrm{O}^{7} \mathrm{O}^{7}$, Neftekumsk, saline floodplain meadow, 23-27.04.2012 (S.A.)

Liocranoeca striata (Kulczyński, 1881)

RECORDS. Petrovsky District [Titova, Egorova, 1978: Agraecina].

\section{LYCOSIDAE}

Allohogna singoriensis (Laxmann, 1770)

MATERIAL. 2 우, Apanasenkovskoe Distr., Manychskoe Vil., garden, 31.05.2005 (T.Kh.).
Alopecosa cuneata (Clerck, 1758)

MATERIAL. $27 \mathrm{O}^{\top} \mathrm{O}^{\top}, 10$ 우, Neftekumsk, saline floodplain meadow, 23-27.04.2012 (S.A.); $27 \mathrm{O}^{\top} \sigma^{7}, 11$ 우 , s.l., salt-pan with tamarix, 23-27.04.2012 (S.A.)

Alopecosa cursor (Hahn, 1831)

MATERIAL. 3 o' $\sigma^{\prime}$, Neftekumsk Distr., $10 \mathrm{~km} \mathrm{~S}$ of Zaterechnyi Vil., sagebrush-grass steppe, 23-27.04.2012 (S.A.); 16 O $^{7} \sigma^{7}, 1$ o, s.1., dune foothill, 23-27.04.2012 (S.A.); 2 O $^{\top} \sigma^{7}$, Neftekumsk, salt-pan with tamarix, 23-27.04.2012 (S.A.).

Alopecosa farinosa (O. Herman, 1879)

MATERIAL. $1 \sigma^{7}$, Stavropol, 17.04.2000 (T.Kh.); $1 \sigma^{7}, 20 \mathrm{~km}$ NE of Stavropol, Kozhevnikov Vil., steppe, 25.03.2001 (T.Kh.); 3 우우, Izobilnyi, 9.07-8.08.2011 (V.K.)

\section{Alopecosa pulverulenta (Clerck, 1758)}

MATERIAL. 6 우, Izobilnyi, 9.07-8.08.2011 (V.K.); 3 ㅇ, s.1., afforestation of Robinia pseudacacia, 18.07-21.08.2013 (V.K.); 35 우, s.1., 5.07-15.08.2014 (V.K.).

Alopecosa taeniopus (Kulczyński, 1895)

MATERIAL. 3 우, Apanasenkovskoe Distr., Manychskoe Vil., Manych-Gudilo Lake shore, 24.05.2005 (T.Kh.); 5 O$^{7} \sigma^{7}$, Neftekumsk Distr., $10 \mathrm{~km} \mathrm{~S}$ of Zaterechnyi Vil., sagebrush-grass steppe, 23-27.04.2012 (S.A.); $1 \sigma^{7}$, Neftekumsk, salt-pan with tamarix, 23-27.04.2012 (S.A.).

Arctosa leopardus (Sundevall, 1832)

MATERIAL 19 , Neftekumsk Distr., nr. Andrei-Kurgan Vil., $1-10.07 .1987$ (S.A.); $150 \sigma^{\top} \sigma^{7}, 100$ 우, Neftekumsk, saline floodplain meadow, 23-27.04.2012 (S.A.); $300^{7} \sigma^{7}, 13$ +o , s.l., salt-pan with tamarix, 23-27.04.2012 (S.A.).

Aulonia albimana (Walckenaer, 1805) (P.I.).

MATERIAL. 1 + , Zheleznovodsk Distr., Beshtau Mt., 8.09.2013

Caspicosa manytchensis Ponomarev, 2007

RECORDS. Stavropol [Ponomarev, 2007]. 2003

Evippa apsheronica Marusik, Guseinov et Koponen,

MATERIAL. $10^{7}$, Neftekumsk Distr., nr. Andrei-Kurgan Vil., $1-10.07 .1987$ (S.A.)

Hogna radiata (Latreille, 1817)

RECORDS. Zheleznovodsk [Schmidt, 1895: Lycosa radiata var. liguriensis].

MATERIAL. $1 \sigma^{\prime}$, Neftekumsk Distr., nr. Andrei-Kurgan Vil.,

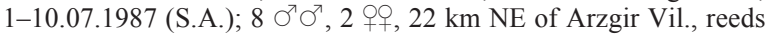
along Chograi Reservoir bank, 14-25.07.1987 (S.A.); $11 \mathrm{O}^{7} \sigma^{7}, 3$ Oㅇ, Izobilnyi, 9.07-8.08.2011 (V.K.); $3 \mathrm{O}^{7} \mathrm{O}^{7}$, s.l., grassy meadow, 18.07-18.08.2013 (V.K.); 3 O $^{7} \mathrm{O}^{7}, 1$ \%, s.l., afforestation of Robinia pseudacacia, 18.07-21.08.2013 (V.K.); $1 \sigma^{7}$, s.1., small holding, 18.07-21.08.2013 (V.K.); 2 O $^{7}$ 丁 $^{7}, 1$ ㅇ, s.1., 5.07-15.08.2014 (V.K.).

Mustelicosa dimidiata (Thorell, 1875)

RECORDS. Manychskoe [Ponomarev et al., 2006].

MATERIAL. $60^{7} \sigma^{7}, 2$ 우, Apanasenkovskoe Distr., Manychskoe Vil., 29.05-2.06.2005 (T.Kh.); 1 ㅇ, Izobilnyi, 5.07-15.08.2014 (V.K.).

Pardosa agrestis (Westring, 1861)

RECORDS. Petrovsky District [Titova, Egorova, 1978: Pardosa monticola, misidentification]. 
MATERIAL. 1 ๆ 17 17 , Neftekumsk Distr., nr. AndreiKurgan Vil., 1-10.07.1987 (S.A.); $14 \sigma^{7} \sigma^{7}, 9$ O+, $22 \mathrm{~km} \mathrm{NE}$ of Arzgir Vil., reeds along Chograi Reservoir bank, 14-25.07.1987 (S.A.); $19 \sigma^{7} \sigma^{7}, 7$ 우, Neftekumsk, saline floodplain meadow, 2327.04.2012 (S.A.); $53 \sigma^{7} \sigma^{7}, 28$ क्ष, s.1., salt-pan with tamarix, $23-$ 27.04.2012 (S.A.); 19 ऽ ऊ', 6 우, Neftekumsk Distr., $10 \mathrm{~km} \mathrm{~S}$ of Zaterechnyi Vil., sagebrush-grass steppe, 23-27.04.2012 (S.A.); 1 $\sigma^{\top}, 3$ 우, near Kravtsovo Lake, 13.06.2000 (T.Kh.); $36 \sigma^{\top} \sigma^{\top}, 21$ +o, Apanasenkovskoe Distr., Manychskoe Vil., Manych-Gudilo Lake shore, 24-30.05.2005 (T.Kh.); $56 \sigma^{7} \sigma^{7}, 15$ 우, s.1., afforesta-

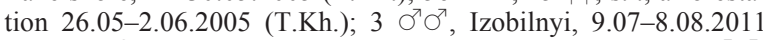
(V.K.); 1 9, s.1., grassy meadow, 18.07-18.08.2013 (V.K.); $4 \sigma^{7} \sigma^{\top}$, 1 , s.1., afforestation of Robinia pseudacacia, 18.07-21.08.2013

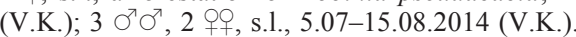

\section{Pardosa italica Tongiorgi, 1966}

MATERIAL. $2 \sigma^{7} \sigma^{7}, 1$ ㅇ, Apanasenkovskoe Distr., Manychskoe Vil., Manych-Gudilo Lake shore, 24-26.05.2005 (T.Kh.); 11 $\sigma^{\top} \sigma^{\top}, 10$ 우, Neftekumsk, saline floodplain meadow, 23-27.04.2012 (S.A.).

Pardosa jaikensis Ponomarev, 2008

MATERIAL. $2 \sigma^{7} \sigma^{7}, 9$ 우, Neftekumsk, saline floodplain meadow, 23-27.04.2012 (S.A.); 12 O $^{7} \sigma^{7}, 9$ क + , s.1., salt-pan with tamarix, 23-27.04.2012 (S.A.).

Pardosa luctinosa Simon, 1876

MATERIAL. $11 \sigma^{\top} \sigma^{7}, 19$ 우, Neftekumsk Distr., nr. AndreiKurgan Vil., 1-10.07.1987 (S.A.); 26 O$^{7} \sigma^{7}, 6$ 우, Neftekumsk, saline floodplain meadow, 23-27.04.2012 (S.A.).

Pardosa lugubris (Walckenaer, 1802)

MATERIAL. 62 우, Izobilnyi, 9.07-8.08.2011 (V.K.); 1 웅 s.1., grassy meadow, 18.07-18.08.2013 (V.K.); 14 우, s.1., afforestation of Robinia pseudacacia, 18.07-21.08.2013 (V.K.); 3 우우, s.l., 5.07-15.08.2014 (V.K.).

Pardosa nebulosa (Thorell, 1872)

RECORDS. Svetlograd, Sengileevskoe Reservoir [Ponomarev et al., 2006].

MATERIAL. 1 \%, Svetlograd, in garden, 1.05.2000 (T.KH.); 1 ㅇ, bank of Sengileevskoe Reservoir, 23.06.2000 (T.Kh.); $1 \sigma^{7}$ Zheleznovodsk Distr., Inozemtsevo, 8.09.2013 (P.I.).

Pardosa paludicola (Clerck, 1758)

MATERIAL. $3 \sigma^{7} \sigma^{7}, 1$, Stavropol, Budarka Mt., 22.03.2001 (T.Kh.)

Pardosa pontica (Thorell, 1875)

RECORDS. Stavropol [Zyuzin, Logunov, 2000].

MATERIAL. 1 ㅇ, Izobilnyi, 9.07-8.08.2011 (V.K.); 7 O $^{7} \mathrm{O}^{7}, 14$ 우, s.1., 5.07-15.08.2014 (V.K.); $1 \mathrm{O}^{7}, 3$ 우, s.1., 5.07-15.08.2014 (V.K.); $1 \mathrm{O}^{\top}, 5$ 우, Neftekumsk, saline floodplain meadow, 2327.04.2012 (S.A.)

Pardosa prativaga (L. Koch, 1870)

MATERIAL. $22 \mathrm{O}^{\top} \mathrm{O}^{\top}, 7$ 우, Neftekumsk, saline floodplain meadow, 23-27.04.2012 (S.A.)

Pirata piraticus (Clerck, 1758)

MATERIAL. $4 O^{7} O^{7}, 3$ 우, Neftekumsk, saline floodplain meadow, 23-27.04.2012 (S.A.)

Piratula hygrophila (Thorell, 1872)

MATERIAL. 2 우, Zheleznovodsk Distr., Beshtau Mt., 2830.07.2005 (A.R.).
Trochosa cachetiensis Mcheidze, 1997

Figs. 10-15.

MATERIAL. $20^{\top} \sigma^{\top}, 2$ ㅇ, Zheleznovodsk Distr., Beshtau Mt., 8.09.2013 (P.I.).

\section{Trochosa robusta (Simon, 1876)}

MATERIAL. 1 , Neftekumsk Distr., nr. Andrei-Kurgan Vil., 1-10.07.1987 (S.A.); 6 O $^{7} \sigma^{7}$, Neftekumsk, saline floodplain meadow, 23-27.04.2012 (S.A.); 4 O $^{7} \sigma^{7}, 3$ 우, s.1., salt-pan with tamariks, 23-27.04.2012 (S.A.); 1 \% , Apanasenkovskoe Distr., Manychskoe Vil., 29.05-2.06.2005 (T.Kh.); 9 O $^{\top} \sigma^{7}, 4$ 우, s.1., 24.05-2.06.2005 (T.Kh.); 1 O $^{\top}$, s.1., 19-30.05.2005 (S.P.); 11 O $^{\top} \mathrm{O}^{\prime}, 11$ 우, Izobilnyi, 9.07-8.08.2011 (V.K.); 4 +9, s.1., grassy meadow, 18.07-18.08.2013 (V.K.); $10^{7}, 4$ 우, s.l., afforestation of Robinia pseudacacia, 18.0721.08.2013 (V.K.); 2 +o, s.1., 5.07-15.08.2014 (V.K.).

Trochosa ruricola (De Geer, 1778)

MATERIAL. $20^{7} \sigma^{7}, 3$ 우, $22 \mathrm{~km}$ NE of Arzgir Vil., reeds along Chograi Reservoir bank, 14-25.07.1987 (S.A.); $3 \mathrm{O}^{7} \mathrm{O}^{7}$, Apanasenkovskoe Distr., Manychskoe Vil., Manych-Gudilo Lake shore, 24-30.05.2005 (T.Kh.); 4 O $^{\top} \sigma^{7}$, s.1., afforestation, 29.05-2.06.2005 (T.Kh.); 1 O , 3 of , Izobilnyi, 9.07-8.08.2011 (V.K.); 1 +, s.1., afforestation of Robinia pseudacacia, 18.07-21.08.2013 (V.K.); 26 오, s.1., 5.07-15.08.2014 (V.K.); $6 \sigma^{7} \sigma^{7}, 1$, Neftekumsk, saline floodplain meadow, 23-27.04.2012 (S.A.); 1 \%, 1 q, Zheleznovodsk Distr., Inozemtsevo, 8.09.2013 (P.I.).

Trochosa terricola Thorell, 1856

RECORDS. Petrovsky District [Titova, Egorova, 1978].

MATERIAL, 1 ․ Stavropol, 17.04.2000 (T.Kh.); 1 \% s.1,

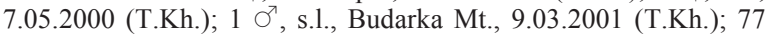
$\sigma^{7} \sigma^{7}, 85$ 우, Izobilnyi, 9.07-8.08.2011 (V.K.); $11 \sigma^{7} \sigma^{7}, 113$ 우, s.1., afforestation of Robinia pseudacacia, 18.07-21.08.2013 (V.K.); 2 Oै $^{\top} 0^{\top}, 8$ 우, s.1., 5.07-15.08.2014 (V.K.).

Xerolycosa miniata (C.L. Koch, 1834)

RECORDS. Beshpagir [Logunov, Penney, 2004].

MATERIAL, 1 , near Kravtsovo Lake, 13.06 .2000 (T.Kh.); $19 \sigma^{7} \sigma^{7}, 6$ 우, Apanasenkovskoe Distr., Manychskoe Vil., 25.052.06.2005 (T.Kh.); $57 \bigcirc^{7} \Im^{7}, 34$ 우, Izobilnyi, 9.07-8.08.2011 (V.K.); $9 \mathrm{O}^{7} \mathrm{O}^{7}, 22$ 우, s.1., grassy meadow, 18.07-18.08.2013 (V.K.); 4 Oొం $^{\top}, 12$ 웅, s.1., $5.07-15.08 .2014$, (V.K.).

Xerolycosa nemoralis (Westring, 1861)

MATERIAL. 2 +o, Izobilnyi, 9.07-8.08.2011 (V.K.).

\section{MITURGIDAE}

Zora armillata Simon, 1878

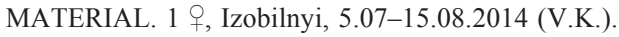

Zora pardalis Simon, 1878

MATERIAL. $1{ }^{\top}$, Apanasenkovskoe Distr., Manychskoe Vil., 25-30.05.2005 (T.Kh.).

Zora silvestris Kulczyński, 1897

MATERIAL. 1 ㅇ, Izobilnyi, 9.07-8.08.2011 (V.K.); 1 ․, s.1., 5.07-15.08.2014 (V.K.).

\section{OXYOPIDAE}

Oxyopes heterophthalmus (Latreille, 1804)

RECORDS. Beshpagir [Logunov, Penney, 2004]; Budennovsk [Esyunin, Tuneva, 2009]. 

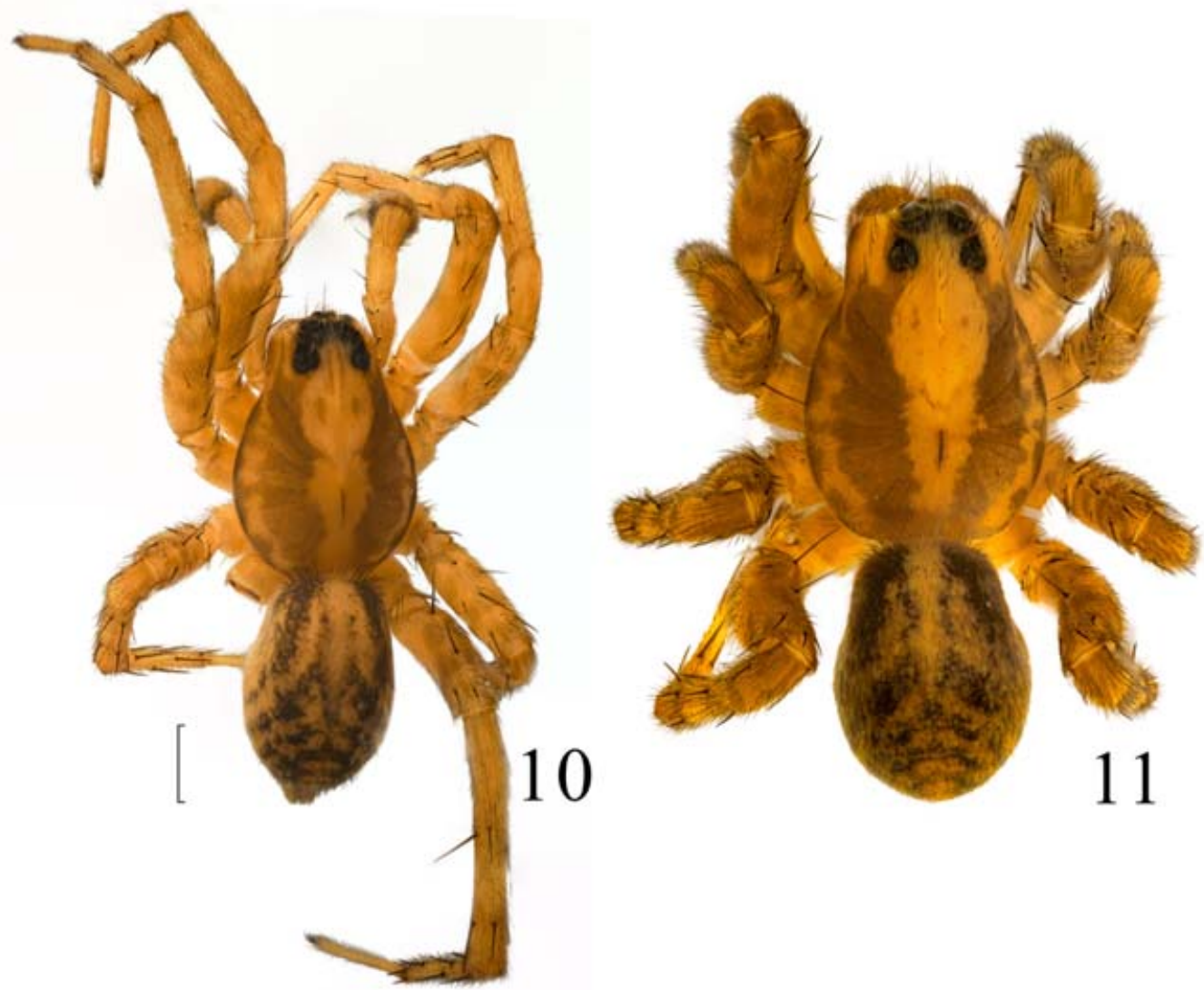

Figs. 10, 11. Trochosa cachetiensis: 10 - male, dorsal view; 11 - female, dorsal view. Scale bars: $1 \mathrm{~mm}$. Рис. 10, 11. Trochosa cachetiensis: 10 - самец, вид сверху; 11 - самка, вид сверху. Масштаб 1 мм.

MATERIAL. $5 \sigma^{7} \sigma^{7}, 5$ 5 , Apanasenkovskoe Distr., Manychskoe Vil., Manych-Gudilo Lake shore, 24-31.05.2005 (T.Kh.); 76 $\sigma^{7} \sigma^{7}, 13$ 우, s.1., steppe, 26-31.05.2005 (T.Kh.); $1 \sigma^{7}$, s.1., saline meadow, 29.05.2005 (T.Kh.); 1 ○, 1 ๆ, s.1., 19-30.05.2005 (S.P.); 1 ㅇ, Medvedskaya Mt. , steppe, 19.06.2005 (E.Kh.).

Oxyopes lineatus Latreille, 1806

RECORDS. Beshpagir [Logunov, Penny, 2004].

MATERIAL. 1 \%, Trunovskoe Distr., Donskoe Vil., steppe, 22.09.2000 (T.Kh.); 2 O $^{7} \sigma^{\top}$, Petrovsky Distr., Gofitskoe Vil., 29.06.2005 (E.Kh.).

Oxyopes xinjiangensis $\mathrm{Hu}$ et $\mathrm{Wu}, 1989$ (E.T.)

MATERIAL. 1 +, Kursky Distr., Sovkhoznyi Vil., 5-6.08.2016

\section{PHILODROMIDAE}

Artanes poecilus (Thorell, 1872)

MATERIAL. 1 +, Izobilnyi, 5.07-15.08.2014 (V.K.).

Philodromus cespitum (Walckenaer, 1802)

RECORDS. Stavropol, Sengileevskoe Reservoir, Manychskoe [Khanov, Ponomarev, 2006].

MATERIAL. 1 9, Izobilnyi, 9.07-8.08.2011 (V.K.); 3 우, s.1., afforestation of Robinia pseudacacia, 18.07-21.08.2013 (V.K.).
Pulhellodromus ruficapillus (Simon, 1885)

RECORDS. Manych-Gudilo [Ponomarev et al., 2006: sub Philodromus glaucinus, misidentification]; Manychskoe [Khanov, Ponomarev, 2006: sub P. glaucinus, misidentification].

Rhysodromus histrio (Latreille, 1819)

RECORDS. Manychskoe [Khanov, Ponomarev, 2006: Philodromus]; Velichaevskoe [Szita, Logunov, 2008: Philodromus].

MATERIAL. 1 +, Apanasenkovskoe Distr., Manychskoe Vil., 19-30.05.2005 (S.P.).

Thanatus arenarius Thorell, 1872

RECORDS. Belye Kopani, Manychskoe [Khanov, Ponomarev, 2006].

MATERIAL. 3 우, Izobilnyi, 9.07-8.08.2011 (V.K.); $210^{7} \sigma^{7}$, 3 우, Neftekumsk Distr., $10 \mathrm{~km} \mathrm{~S}$ of Zaterechnyi Vil., sagebrushgrass steppe, 23-27.04.2012 (S.A.); $10^{7}$, s.1., dune foothill, 2327.04.2012 (S.A.); 9 O $^{2} \sigma^{\top}, 1$, Neftekumsk, salt-pan with tamariks, 23-27.04.2012 (S.A.).

Thanatus atratus Simon, 1875

MATERIAL. $10^{7}$, Neftekumsk Distr., nr. Andrei-Kurgan Vil., 1-10.07.1987 (S.A.). 

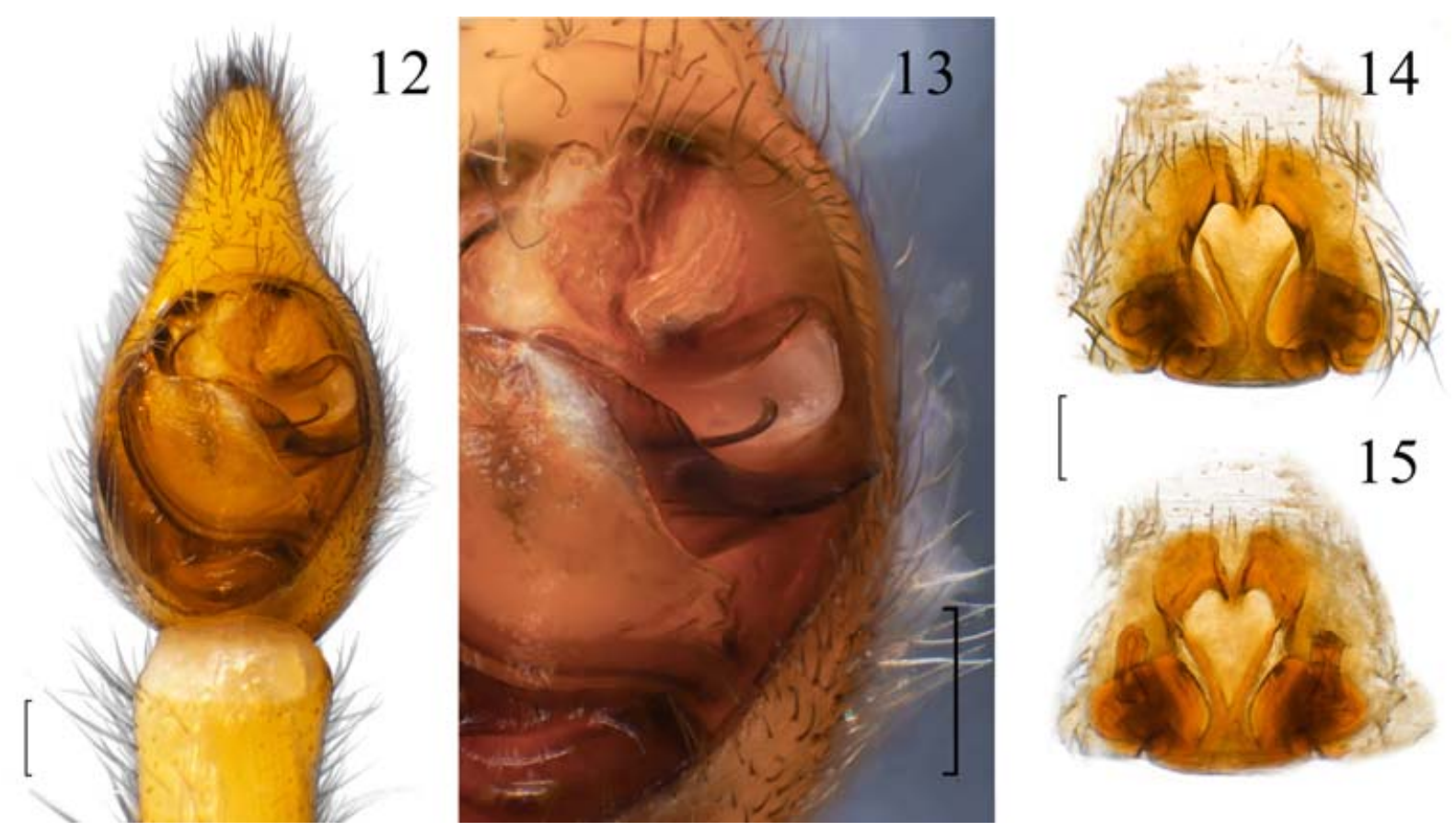

Figs. 12-15. Trochosa cachetiensis: 12 - male palp, ventral view; 13 - part of bulbus, ventral view; 14 - epigyne, ventral view; 15 - epigyne, dorsal view. Scale bars: $0.2 \mathrm{~mm}$.

Рис. 12-15. Trochosa cachetiensis: 12 — пальпа самца, вид снизу; 13 - часть бульбуса, вид снизу; 14 - эпигина, вид снизу; 15 - эпигина, вид сверху. Масштаб 0,2 мм.

Thanatus imbecillus L. Koch, 1878

MATERIAL. $2 \sigma^{7} \sigma^{7}$, Neftekumsk Distr., $10 \mathrm{~km} \mathrm{~S}$ of Zaterechnyi Vil., sagebrush-grass steppe, 23-27.04.2012 (S.A.); 13 O $^{7} O^{7}$, s.1., dune foothill, 23-27.04.2012 (S.A.); 1 +, Neftekumsk, saltpan with tamarix, 23-27.04.2012 (S.A.).

Thanatus oblongiusculus (Lucas, 1846)

RECORDS. Manychskoe [Ponomarev et al., 2006; Khanov, Ponomarev, 2006: in both sub Paratibellus].

MATERIAL. $1+20 \mathrm{~km} \mathrm{~S}$ of Stavropol, Strizhament Mt., 18.07.2013 (E.T.)

Tibellus oblongus (Walckenaer, 1802)

RECORDS. Petrovsky district [Titova, Egorova, 1978]; Sengileevskoe Reservoir, Manychskoe, Prietoksky [Khanov, Ponomarev, 2006].

MATERIAL. 2 우, Izobilnyi, afforestation of Robinia pseudacacia, 18.07-21.08.2013 (V.K.); 1 +, s.1., 5.07-15.08.2014 (V.K.).

\section{PHOLCIDAE}

Pholcus ponticus Thorell, 1875

RECORDS. Beshpagir [Logunov, Penney, 2004]; Manychskoe [Ponomarev et al., 2006].

MATERIAL. $20^{\top} \sigma^{7}, 4$ O, , Apanasenkovskoe Distr., Manychskoe Vil., apartment building, 3.06.2005 (T.Kh.); 2 우, Zheleznovodsk Distr., Inozemtsevo, 8.09.2013 (P.I.); 1 O', Izobilnyi, apartment building, 18.07-20.08.2013 (V.K.).

\section{PHRUROLITHIDAE}

Phrurolithus festivus (C.L. Koch, 1835)

RECORDS. Beshpagir [Logunov, Penney, 2004].

\section{PISAURIDAE}

Pisaura mirabilis (Clerck, 1758)

RECORDS. Petrovsky District [Titova, Egorova, 1978]; Beshpagir [Logunov, Penney, 2004].

Pisaura novicia (L. Koch, 1878)

MATERIAL. 1 \% , Stavropol, Chlinskiy forest, 7.05.2000 (T.Kh.); 3 O $^{7} \sigma^{\top}, 4$ 우, Apanasenkovskoe Distr., Manychskoe Vil., Manych-Gudilo Lake shore, 24-31.05.2005 (T.Kh.); 6 O $^{7} \sigma^{7}$, s.1., afforestation, 29.05-2.06.2005 (T.Kh.).

\section{SALTICIDAE}

Aelurillus laniger Logunov et Marusik, 2000

MATERIAL. $10^{7}$, Apanasenkovskoe Distr., Manychskoe Vil., 25-30.05.2005 (T.Kh.)

Aelurillus v-insignitus (Clerck, 1758)

MATERIAL. $3 \sigma^{7} \sigma^{7}, 1$, Apanasenkovskoe Distr., Manychskoe Vil., steppe, 25-31.05.2005 (T.Kh.); $3 \sigma^{7} \sigma^{7}$, s.l., afforestation, 26.05-2.06.2005 (T.Kh.); 6 + 웅, Izobilnyi, 9.07-8.08.2011 (V.K.); $20^{7} \sigma^{7}$, Neftekumsk Distr., $10 \mathrm{~km} \mathrm{~S}$ of Zaterechnyi Vil., sagebrushgrass steppe, 23-27.04.2012 (S.A.); 3 O $^{7} \sigma^{7}$, s.1., dune foothill, 2327.04.2012 (S.A.).

Ballus chalybeius (Walckenaer, 1802)

MATERIAL. 1 +, Apanasenkovskoe Distr., Manychskoe Vil., Manych-Gudilo Lake shore, 24.05.2005 (T.Kh.).

Carrhotus xanthogramma (Latreille, 1819)

RECORDS. Stavropol [Logunov, Guseinov, 2002]; Essentuki [Logunov, 2015]. 
Euophrys frontalis (Walckenaer, 1802)

MATERIAL. $1 \sigma^{7}$, Apanasenkovskoe Distr., Manychskoe Vil., 25-30.05.2005 (T.Kh.); 2 오, Izobilnyi, 9.07-8.08.2011 (V.K.); 3 어, s.l., afforestation of Robinia pseudacacia, 18.07-21.08.2013 (V.K.).

Evarcha arcuata (Clerck, 1758)

RECORDS. Beshpagir [Logunov, Penny, 2004]; Essentuki [Logunov, 2015].

MATERIAL. $1 \bigcirc^{7}$, Sengileevskoe Reservoir, steppe, 11.06.2000 (T.Kh.); 1 O$^{7}$, Izobilnyi, 9.07-8.08.2011 (V.K.); 1 O', 2 우, s.1., afforestation of Robinia pseudacacia, 18.07-21.08.2013 (V.K.); 1 ơ, s.l., 5.07-15.08.2014 (V.K.); 1 Ơ, Zheleznovodsk Distr., Beshtau Mt., 25-27.07.2015 (E.T.)

Heliophanus auratus C.L. Koch, 1835

RECORDS. Beshpagir [Logunov, Penney, 2004; Logunov, 2015].

MATERIAL. $1 \sigma^{7}$, Apanasenkovskoe Distr., Manychskoe Vil., saline meadow, 29.05.2005 (T.Kh.); $1 \mathrm{O}^{\top}, 1$ + , s.l., afforestation, 26.05-2.06.2005 (T.Kh.).

Heliophanus cupreus (Walckenaer, 1802)

RECORDS. Beshpagir [Logunov, Penney, 2004].

MATERIAL. $1 \sigma^{7}$, Stavropol, Tamanskiy Forest, 30.04.2000 (T.Kh.); 1 , Pyatigorsk, N slope of Mashuk Mt., $500 \mathrm{~m}$ a.s.l., forest, 27.07.2008 (A.Z.).

Heliophanus flavipes (Hahn, 1832)

MATERIAL. $1 \sigma^{7}$, Stavropol, Russian Forest, Besputskaya Clearing Botanical Park, 23.06.2000 (T.Kh.); 1 \%, Apanasenkovskoe Distr., Manychskoe Vil., afforestation, 25.05.2005 (T.Kh.); $5 \mathrm{O}^{7} \mathrm{O}^{7}, 5$ 우, s.1., afforestation, 25.05-2.06.2005 (T.Kh.); 1 , Stavropol, 3.07.2005 (E.Kh.).

Heliophanus koktas Logunov, 1992

RECORDS. Manychskoe [Ponomarev et al., 2008]. MATERIAL. 1 , Apanasenkovskoe Distr., Manychskoe Vil., pasture, 26.05.2005 (T.Kh.)

Heliophanus patagiatus Thorell, 1875

MATERIAL. $10^{7}$, Apanasenkovskoe Distr., Manychskoe Vil., afforestation, 29.05-2.06.2005 (T.Kh.).

Myrmarachne formicaria (De Geer, 1778)

MATERIAL. $1 \sigma^{7}$, Izobilnyi, afforestation of Robinia pseudacacia, 18.07-21.08.2013 (V.K.)

Neon rayi (Simon, 1875)

MATERIAL. 1 +, Neftekumsk Distr., nr. Andrei-Kurgan Vil., $1-10.07 .1987$ (S.A.).

Pellenes allegrii Caporiacco, 1935

MATERIAL. 1 +, Neftekumsk Distr., nr. Andrei-Kurgan Vil., 1-10.07.1987 (S.A.); 1 O$^{\top}, 1$ ㅇ, Neftekumsk, saline floodplain meadow, 23-27.04.2012 (S.A.).

Pellenes nigrociliatus (Simon in L. Koch, 1875)

MATERIAL. 1 ㅇ, Apanasenkovskoe Distr., Manychskoe Vil., afforestation, 29.05-2.06.2005 (T.Kh.).

Pellenes seriatus (Thorell, 1875)

MATERIAL. $1 \mathrm{O}^{7}$, Apanasenkovskoe Distr., Manychskoe Vil., steppe, 31.05.2005 (T.Kh.); 2 Oొ $^{7}, 2$ 우, Neftekumsk, saline floodplain meadow, 23-27.04.2012 (S.A.); 3 ○ $^{7} \sigma^{7}$, s.1., salt-pan with tamarix, 23-27.04.2012 (S.A.); 1 O $^{\prime}$, Neftekumsk Distr., $10 \mathrm{~km} \mathrm{~S}$ of Zaterechnyi Vil., sagebrush-grass steppe, 23-27.04.2012 (S.A.).
Phlegra fasciata (Hahn, 1826)

MATERIAL. 4 O $^{7}, 1$, , Izobilnyi, 9.07-8.08.2011 (V.K.); 2 우, s.1., grassy meadow, 18.07-18.08.2013 (V.K.); 1 o', 1 ㅇ, s.l., afforestation of Robinia pseudacacia, 18.07-21.08.2013 (V.K.); 5 $\mathrm{O}^{7} \mathrm{O}^{7}, 3$ 우, s.1., $5.07-15.08 .2014$ (V.K.).

Philaeus chrysops (Poda, 1761)

RECORDS. Pyatigorsk [Verzhbitsky, 1902].

MATERIAL. 1 +, Apanasenkovskoe Distr., Manychskoe Vil., trampled steppe, 26.05.2005 (T.Kh.)

Pseudeuophrys erratica (Walckenaer, 1825)

RECORDS. Pyatigorsk [Logunov, 1998].

MATERIAL. $1 \sigma^{7}$, Stavropol, Chlinskiy forest, 7.05.2000 (T.Kh.); 1 , Zheleznovodsk Distr., Beshtau Mt., 8.09.2013 (P.I.).

Pseudeuophrys obsoleta (Simon, 1868)

MATERIAL. $10^{7}$, Apanasenkovskoe Distr., Manychskoe Vil., afforestation, 26.05.2005 (T.Kh.)

Pseudicius encarpatus (Walckenaer, 1802)

RECORDS. Stavropol [Logunov, Guseinov, 2002].

MATERIAL. 1 \%, Neftekumsk, salt-pan with tamarix, 2327.04.2012 (S.A.).

Salticus tricinctus (C.L. Koch, 1846)

MATERIAL. $1 \sigma^{7}$, Apanasenkovskoe Distr., Manychskoe Vil., Manych-Gudilo Lake shore, 24.05.2005 (T.Kh.); $2 \sigma^{7} \sigma^{7}, 2$ 오, s.1., afforestation, 26.05-2.06.2005 (T.Kh.).

Sitticus dzieduszyckii (L. Koch, 1870)

RECORDS. Stavropol [Logunov, Guseinov, 2002].

MATERIAL. 2 o+ , Trunovskoe Distr., Donskoe Vil., steep river bank, 22.09.2000 (T.Kh.)

Sitticus penicillatus (Simon, 1875)

MATERIAL. $1 \sigma^{7}$, Izobilnyi, 9.07-8.08.2011 (V.K.); 1 ○', s.l., 5.07-15.08.2014 (V.K.).

Sitticus zimmermanni Simon, 1877

MATERIAL. $2 \sigma^{7} \sigma^{7}, 1$, Izobilnyi, 9.07-8.08.2011 (V.K.); 2 $\mathrm{O}^{7} \mathrm{O}^{\mathrm{T}}$, s.1., afforestation of Robinia pseudacacia, 18.07-21.08.2013 (V.K.); 2 ○ $^{7}, 2$ 우, s.1., 5.07-15.08.2014 (V.K.).

Talavera petrensis (C.L. Koch, 1837)

MATERIAL. $1 \mathrm{O}^{7}$, Apanasenkovskoe Distr., Manychskoe Vil., afforestation, 29.05-2.06.2005 (T.Kh.).

\section{SPARASSIDAE}

Micrommata virescens (Clerck, 1758)

MATERIAL. 1 juv., Sengileevskoe Reservoir, grassy steppe, 17.09.2000 (T.Kh.)

\section{TETRAGNATHIDAE}

Metellina merianae (Scopoli, 1763)

MATERIAL. $1 \sigma^{7}$, Zheleznovodsk Distr., Inozemtsevo Vil., 89.09.2013 (P.I.).

Pachygnatha clercki Sundevall, 1823

MATERIAL. $1 \sigma^{7}$, Apanasenkovskoe Distr., Manychskoe Vil., Manych-Gudilo Lake shore, 24.05.2005 (T.Kh.); $2 O^{7} \sigma^{7}$, Neftekumsk, saline floodplain meadow, 23-27.04.2012 (S.A.). 
Pachygnatha degeeri Sundevall, 1830

MATERIAL. $10^{7}$, Apanasenkovskoe Distr., Manychskoe Vil., 25-30.05.2005 (T.Kh.); 1 O , 2 OO+, s.l., afforestation, 29.052.06.2005 (T.Kh.); $9 \mathrm{O}^{7} \sigma^{7}, 18$ 우, Neftekumsk, saline floodplain meadow, 23-27.04.2012 (S.A.); 1 + , s.l., salt-pan with tamariks, 23-27.04.2012 (S.A.); 4 우, Izobilnyi, 5.07-15.08.2014 (V.K.).

Tetragnatha extensa (Linnaeus, 1758) 1978].

RECORDS. Petrovsky District [Titova, Egorova,

MATERIAL. $50^{\top} \sigma^{7}, 7$ 우, Apanasenkovskoe Distr., Manychskoe Vil., Manych-Gudilo Lake shore, 26-31.05.2005 (T.Kh.).

Tetragnatha pinicola $\mathrm{L}$. Koch, 1870

MATERIAL. 1 , Sengileevskoe Reservoir, 11.06 .2000 (T.Kh.); $1 \mathrm{O}^{\mathrm{T}}, 4$ 우, Apanasenkovskoe Distr., Manychskoe Vil., ManychGudilo Lake shore, 24-26.05.2005 (T.Kh.).

\section{THERIDIIDAE}

Asagena phalerata (Panzer, 1801)

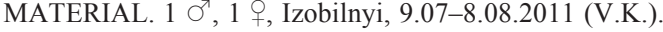

Dipoena melanogaster (C.L. Koch, 1837)

MATERIAL. 1 , Pyatigorsk, S slope of Mashuk Mt., $600 \mathrm{~m}$ a.s.1., forest edge, 27.07.2008 (A.Z.).

Enoplognatha latimana Hippa et Oksala, 1982

RECORDS. Zheleznovodsk [Ponomarev, Mikhailov, 2007].

MATERIAL. 3 Oᄋ, Pyatigorsk, S slope of Mashuk Mt., $600 \mathrm{~m}$ a.s.l., forest edge, 27.07.2008 (A.Z.).

Enoplognatha ovata (Clerck, 1758)

RECORDS. Beshpagir [Logunov, Penney, 2004].

MATERIAL. 2 우, Pyatigorsk, S slope of Mashuk Mt., $600 \mathrm{~m}$ a.s.1., forest edge, 27.07.2008 (A.Z.).

Enoplognatha thoracica (Hahn, 1833)

MATERIAL. $1 \sigma^{7}, 1$, Apanasenkovskoe Distr., Manychskoe Vil., afforestation, 26.05.2005 (T.Kh.); $1 \sigma^{7}$, s.1., steppe, 31.05.2005 (T.Kh.); 1 +, Izobilnyi, 9.07-8.08.2011 (V.K.).

Episinus truncatus Latreille, 1809

RECORDS. Beshpagir [Logunov, Penney, 2004].

MATERIAL. $90^{7} \sigma^{7}$, 13 우, Izobilnyi, 9.07-8.08.2011 (V.K.); 1 9, s.l., afforestation of Robinia pseudacacia, 18.07-21.08.2013 (V.K.).

Euryopis saukea Levi, 1951

RECORDS. Beshpagir [Logunov, Penney, 2004].

MATERIAL. $1 \sigma^{7}$, Apanasenkovskoe Distr., Manychskoe Vil., Manych-Gudilo Lake shore, 24.05.2005 (T.Kh.); 2 O$^{7} \sigma^{7}$, s.l., steppe, 26.05.2005 (T.Kh.).

Heterotheridion nigrovariegatum (Simon, 1873)

MATERIAL. 3 우, Pyatigorsk, N slope of Mashuk Mt., $500 \mathrm{~m}$ a.s.l., forest, 27.07.2008, (A.Z.).

Kochiura aulica (C.L. Koch, 1838) (E.T.).
Latrodectus tredecimguttatus (P. Rossi, 1790)

RECORDS. Stavropol Province [Köppen, 1881].

MATERIAL. 1 + $22 \mathrm{~km}$ NE of Arzgir Vil., reeds along Chograi Reservoir bank, 14-25.07.1987 (S.A.).

Parasteatoda tepidariorum (C.L. Koch, 1841)

RECORDS. Beshpagir [Logunov, Penney, 2004: sub Achaearanea]; Stavropol [Ponomarev et al., 2006: sub Achaearanea].

MATERIAL. 10,1 , Stavropol, Botanical garden, in greenhouse, 7.06.2000 (T.Kh.); 1 9, Zheleznovodsk Distr., Inozemtsevo Vil., 8-9.09.2013 (P.I.).

Phylloneta impressa (L. Koch, 1881)

RECORDS. Beshpagir [Logunov, Penney, 2004: sub Theridion].

MATERIAL. 1 q, Molochnyi Vil., 17.09 .2000 (T.Kh.); 1 ㅇ, Sengileevskoe Reservoir, grassy steppe, 17.09.2000 (T.Kh.); 1 O", Apanasenkovskoe Distr., Manychskoe Vil., afforestation, 25.05.2005 (T.Kh.); 6 O$^{7} \sigma^{7}, 3$ +, , s.l., Manych-Gudilo Lake shore, 26-31.05.2005 (T.Kh.); $10^{7}, 1$ + , s.l., steppe, 31.05.2005 (T.Kh.); $1 \mathrm{O}^{7}$, Petrovsky Distr., Gofitskoe Vil., steppe, 18.06.2005 (E.Kh.).

Robertus golovatchi Eskov, 1987 (P.I.).

MATERIAL. 1 + , Zheleznovodsk Distr., Beshtau Mt., 8.09.2013

Robertus heydemanni Wiehle, 1965

RECORDS. Manychskoe [Ponomarev et al., 2006].

MATERIAL. $1 \sigma^{7}$, Apanasenkovskoe Distr., Manychskoe Vil., Manych-Gudilo Lake shore, 26.05.2005 (T.Kh.).

Robertus lividus (Blackwall, 1836)

MATERIAL. 2 O $^{7}$, Izobilnyi, 9.07-8.08.2011 (V.K.); $1 \sigma^{7}$, s.1., afforestation of Robinia pseudacacia, 18.07-21.08.2013 (V.K.); 1 ơ, s.1., 5.07-15.08.2014 (V.K.).

Steatoda albomaculata (De Geer, 1778)

MATERIAL. $1 \sigma^{7}$, Izobilnyi, 9.07-8.08.2011 (V.K.); 1 o', Neftekumsk Distr., $10 \mathrm{~km} \mathrm{~S}$ of Zaterechnyi Vil., dune foothill, 2327.04.2012 (S.A.).

Steatoda bipunctata (Linnaeus, 1758)

MATERIAL. $1 \sigma^{7}, 3$ 우, Zheleznovodsk Distr., Inozemtsevo Vil., 8.09.2013 (P.I.)

Steatoda castanea (Clerck, 1758)

RECORDS. Beshpagir [Logunov, Penney, 2004].

MATERIAL. $1 \sigma^{7}$, Stavropol, inside an apartment house, 17.06.2000 (T.Kh.); 1 +, Mikhailovsk, 27.09.2000 (T.Kh.); 1 \%, Apanasenkovskoe Distr., Manychskoe Vil., inside house, 3.06.2005 (T.Kh.).

Steatoda paykulliana (Walckenaer, 1806)

MATERIAL. 1 , Apanasenkovskoe Distr., Manychskoe Vil., garden, 31.05.2005 (T.Kh.).

Steatoda triangulosa (Walckenaer, 1802)

RECORDS. Beshpagir [Logunov, Penney, 2004].

MATERIAL. 1 +, Zheleznovodsk Distr., Inozemtsevo Vil., 89.2013 (P.I.).

Theridion uhligi Martin, 1974

RECORDS. Manychskoe [Ponomarev, 2008b]. 


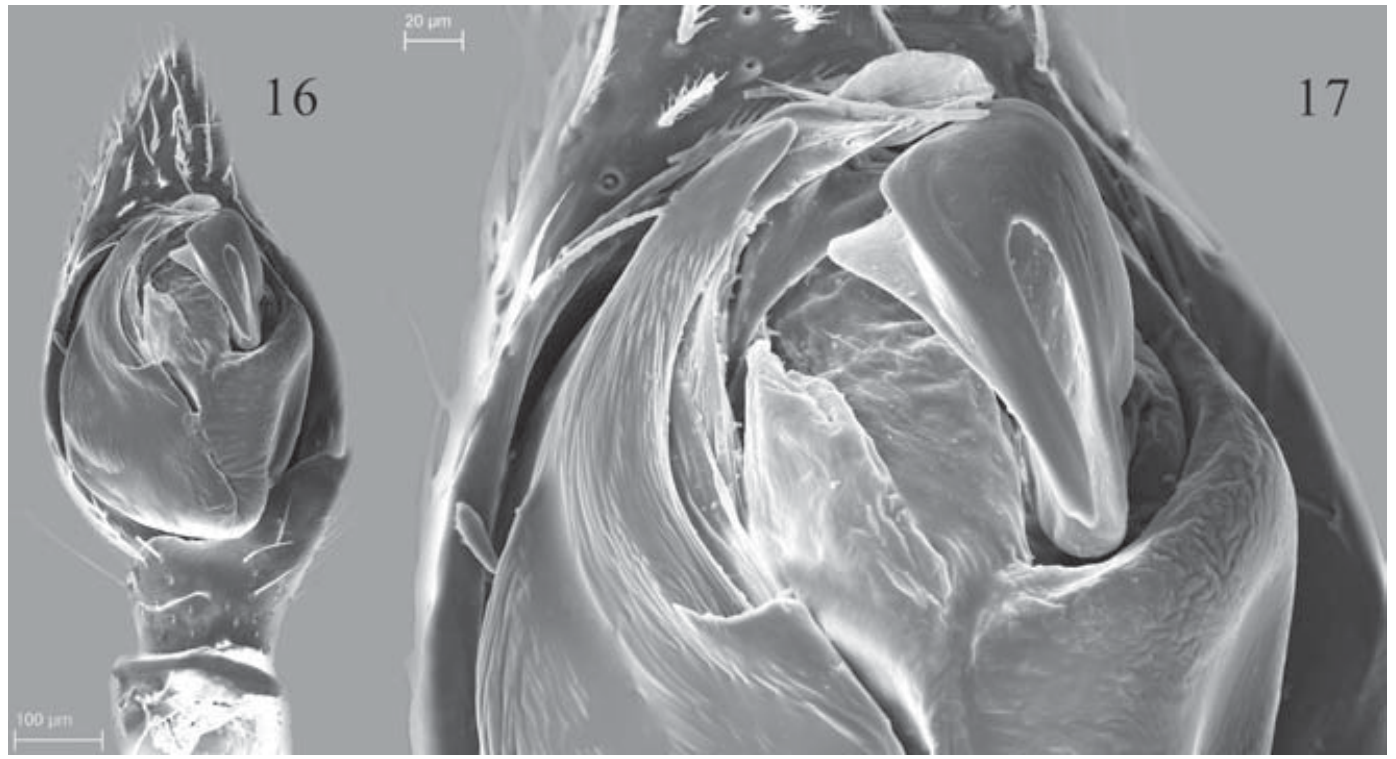

Figs. 16, 17. Zodarion rubidium: 16 - male palp, ventral view; 17 - distal part of bulbus, ventral, view.

Рис. 16, 17. Zodarion rubidium: 16 - пальпа самца, вид снизу; 17 - дистальная часть бульбуса, вид снизу.

Theridion varians (Hahn, 1833)

MATERIAL. $1 \sigma^{\top}$, Stavropol, Botanical Garden, inside greenhouse, 7.06.2000 (T.Kh.).

\section{THOMISIDAE}

Cosyptila guseinovorum Marusik et Kovblyuk in Marusik, Lthtinen et Kovblyuk, 2005

RECORDS. Beshpagir [Logunov, Penney, 2004: sub Ozyptila sp.; Marusik et al., 2005]; Stavropol [Ponomarev et al., 2006; Khanov, Ponomarev, 2006: both as Ozyptila blackwalli, misidentification].

Ebrechtella tricuspidata (Fabricius, 1775)

RECORDS. Stavropol, Sengileevskoe Reservoir, Donskoe, Manychskoe, Mikhailovsk, Prietoksky [Khanov, Ponomarev, 2006: Misumenops].

MATERIAL. 1 +, Izobilnyi, 5.07-15.08.2014 (V.K.)

Heriaeus oblongus Simon, 1918

RECORDS. Manychskoe [Khanov, Ponomarev, 2006].

MATERIAL. 1 +, Zheleznovodsk Distr., Beshtau Mt., 25 27.07.2015 (E.T.).

Misumena vatia (Clerck, 1758)

RECORDS. Stavropol [Lukianov, 1897]; Beshpagir [Logunov, Penney, 2004]; Stavropol, Gofitskoe, Mikhailovsk [Khanov, Ponomarev, 2006].

MATERIAL. 3 우, Zheleznovodsk Distr., Beshtau Mt., 2527.07.2015 (E.T.)

Ozyptila atomaria (Panzer, 1801)

MATERIAL. 2 우, Izobilnyi, afforestation of Robinia pseudacacia, 18.07-21.08.2013 (V.K.); 2 우, s.1., 5.07-15.08.2014 (V.K.).

Ozyptila praticola (C.L. Koch, 1837)

RECORDS. Beshpagir [Logunov, Penney, 2004]; Stavropol [Khanov, Ponomarev, 2006].
MATERIAL. 3 O$^{7} \sigma^{7}, 28$ 우, Izobilnyi, 9.07-8.08.2011 (V.K.); 13 오, s.l., afforestation of Robinia pseudacacia, 18.07-21.08.2013 (V.K.); 2 우, s.1., 5.07-15.08.2014 (V.K.).

Ozyptila scabricula (Westring, 1851) 2006]

RECORDS. Manychskoe [Khanov, Ponomarev,

MATERIAL. 1 + , Izobilnyi, 9.07-8.08.2011 (V.K.); 2 우, s.l., grassy meadow, 18.07-18.08.2013 (V.K.); $1 \sigma^{7}$, Neftekumsk Distr., $10 \mathrm{~km} \mathrm{~S}$ of Zaterechnyi Vil., dune foothill, 23-27.04.2012 (S.A.); 1 $\mathrm{O}^{7}$, Neftekumsk, salt-pan with tamarix, 23-27.04.2012 (S.A.).

Ozyptila simplex (O. Pickard-Cambridge, 1862)

MATERIAL. 2 +q, Izobilnyi, 9.07-8.08.2011 (V.K.).

Pistius truncatus (Pallas, 1772) 2006].

RECORDS. Kravtsovo Lake [Khanov, Ponomarev,

Runcinia grammica (C.L. Koch, 1837)

RECORDS. Stavropol [Khanov, Ponomarev, 2006: Runcinia lateralis].

MATERIAL. 1 \%, Pyatigorsk, S slope of Mashuk Mt., $600 \mathrm{~m}$ a.s.l., forest edge, 27.07.2008 (A.Z.); 1 +, Izobilnyi, 9.07-8.08.2011

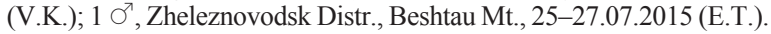

Synema globosum (Fabricius, 1775)

RECORDS. Beshpagir [Logunov, Penney, 2004]; Sengileevskoe Reservoir, Gofitskoe, Prietoksky [Khanov, Ponomarev, 2006].

Thomisus onustus Walckenaer, 1805

RECORDS. Beshpagir [Logunov, Penney, 2004]; Belye Kopani, Manychskoe, Gofitskoe [Khanov, Ponomarev, 2006: Thomisus albus]; Zheleznovodsk [Ponomarev, Mikhailov, 2007: Thomisus albus].

Xysticus acerbus Thorell, 1872

MATERIAL. $1 \mathrm{O}^{\top}$, Neftekumsk Distr., $10 \mathrm{~km} \mathrm{~S}$ of Zaterechnyi Vil., dune foothill, 23-27.04.2012 (S.A.); 1 +, Neftekumsk, saline floodplain meadow, 23-27.04.2012 (S.A.). 
Table 1. List of spider families found in Stavropol Province and their species richness. Таблица 1. Список семейств пауков, выявленных в Ставропольском крае и их видовое богатство.

\begin{tabular}{|c|c|c|c|}
\hline Families & $\begin{array}{l}\text { Total number of species } \\
\text { recorded }\end{array}$ & Our records & Literature records \\
\hline 1. Agelenidae & 9 & 8 & 3 \\
\hline 2. Araneidae & 15 & 13 & 8 \\
\hline 3. Atypidae & 1 & - & 1 \\
\hline 4. Clubionidae & 8 & 3 & 7 \\
\hline 5. Dictynidae & 4 & 4 & - \\
\hline 6. Dysderidae & 3 & 1 & 2 \\
\hline 7. Eresidae & 1 & 1 & 1 \\
\hline 8. Eutichuridae & 7 & 7 & - \\
\hline 9. Gnaphosidae & 51 & 47 & 13 \\
\hline 10. Linyphiidae & 33 & 18 & 19 \\
\hline 11. Liocranidae & 4 & 3 & 1 \\
\hline 12. Lycosidae & 29 & 28 & 8 \\
\hline 13. Miturgidae & 3 & 3 & - \\
\hline 14. Oxyopidae & 3 & 3 & 2 \\
\hline 15. Philodromidae & 9 & 8 & 6 \\
\hline 16. Pholcidae & 1 & 1 & 1 \\
\hline 17. Phrurolithidae & 1 & - & 1 \\
\hline 18. Pisauridae & 2 & 1 & 1 \\
\hline 19. Salticidae & 26 & 25 & 9 \\
\hline 20. Sparassidae & 1 & 1 & - \\
\hline 21. Tetragnathidae & 5 & 5 & 1 \\
\hline 22. Theridiidae & 22 & 21 & 11 \\
\hline 23. Thomisidae & 22 & 16 & 15 \\
\hline 24. Titanoecidae & 2 & 2 & - \\
\hline 25. Uloboridae & 1 & 1 & 1 \\
\hline 26. Zodariidae & 2 & 2 & - \\
\hline Total, species & 265 & 222 & 111 \\
\hline
\end{tabular}

Xysticus bifasciatus C.L. Koch, 1837

MATERIAL. 1 +, Zheleznovodsk Distr., Beshtau Mt., 2527.07.2015 (E.T.).

Xysticus cristatus (Clerck, 1758) 2006].

RECORDS. Manychskoe [Khanov, Ponomarev,

MATERIAL. $2 \sigma^{7} \sigma^{7}$, Neftekumsk, saline floodplain meadow, 23-27.04.2012 (S.A.).

Xysticus kochi Thorell, 1872

RECORDS. Petrovsky District [Titova, Egorova, 1978]; Belye Kopani, Manychskoe, Prietoksky [Khanov, Ponomarev, 2006].

MATERIAL. $1 \bigcirc^{7}$, Stavropol, Tamanskiy Forest, 15.06.2000 (T.Kh.); 1 \%, bank of Sengileevskoe Reservoir, 23.06.2000 (T.Kh.); 2 오, Izobilnyi, 9.07-8.08.2011 (V.K.); $7 \mathrm{O}^{7} \mathrm{O}^{7}$, Neftekumsk, saline floodplain meadow, 23-27.04.2012 (S.A.); 5 O $^{7} \sigma^{7}$, s.l., salt-pan with tamarix, 23-27.04.2012 (S.A.); $18 \mathrm{O}^{7} \sigma^{7}, 1$ o, Neftekumsk Distr., $10 \mathrm{~km} \mathrm{~S}$ of Zaterechnyi Vil., sagebrush-grass steppe, 2327.04.2012 (S.A.); 1 O$^{7}$, s.1., dune foothill, 23-27.04.2012 (S.A.).
Xysticus laetus Thorell, 1875

RECORDS. Nezlobnaya [Esyunin, Òóíåâà, 2012].

MATERIAL. 2 우, Izobilnyi, 9.07-8.08.2011 (V.K.); $17 \mathrm{O}^{71} \mathrm{O}^{7}$, 7 우, Neftekumsk, saline floodplain meadow, 23-27.04.2012 (S.A.); $4 \sigma^{7} \sigma^{7}$, s.1., salt-pan with tamarix, 23-27.04.2012 (S.A.); $1 \mathrm{O}^{\prime 2}$, Neftekumsk Distr., $10 \mathrm{~km} \mathrm{~S}$ of Zaterechnyi Vil., sagebrush-grass steppe, 23-27.04.2012 (S.A.).

Xysticus lanio C.L. Koch, 1845

RECORDS. Stavropol [Ponomarev et al., 2006; Khanov, Ponomarev, 2006].

Xysticus luctator L. Koch, 1870

MATERIAL. $238 \sigma^{7} \sigma^{7}, 12$ क⿱中, Izobilnyi, 9.07-8.08.2011 (V.K.); $1 \sigma^{7}, 3$ 우, s.l., afforestation of Robinia pseudacacia, $18.07-21.08 .2013$ (V.K.).

Xysticus ninnii (Thorell, 1872)

MATERIAL. 1 \% , Zheleznovodsk Distr., Beshtau Mt., 2527.07.2015 (E.T.). 
Xysticus robustus (Hahn, 1832)

MATERIAL. $60^{7} \sigma^{7}, 1$ \%, Izobilnyi, 9.07-8.08.2011 (V.K.); 11 $\mathrm{O}^{7} \mathrm{O}^{7}, 3$ 우, s.1., afforestation of Robinia pseudacacia, 18.07-

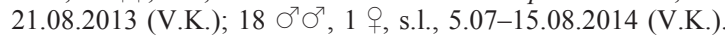

Xysticus striatipes L. Koch, 1870

RECORDS. Stavropol, Sengileevskoe Reservoir, Donskoe [Khanov, Ponomarev, 2006].

\section{TITANOECIDAE}

Nurscia albomaculata (Lucas, 1846)

MATERIAL. $7 \bigcirc^{7} \sigma^{7}$, Neftekumsk Distr., nr. Andrei-Kurgan Vil., 1-10.07.1987 (S.A.); 18 ○ $^{7}, 4$ 우, $22 \mathrm{~km} \mathrm{NE}$ of Arzgir Vil., reeds along Chograi Reservoir bank, 14-25.07.1987 (S.A.).

Titanoeca schineri L. Koch, 1872

MATERIAL. $20^{7} \sigma^{7}$, Apanasenkovskoe Distr., Manychskoe Vil., afforestation, 26.05-2.06.2005 (T.Kh.); $87 \sigma^{7} \sigma^{7}, 1$ \% , Izobilnyi, 9.07-8.08.2011 (V.K.); $13 \sigma^{7} \sigma^{7}, 2$ 우, s.1., afforestation of Robinia pseudacacia, 18.07-21.08.2013 (V.K.); 8 O$^{7} \sigma^{7}, 1$ + , s.l., 5.07-15.08.2014 (V.K.).

\section{ULOBORIDAE}

Uloborus walckenaerius Latreille, 1806

RECORDS. Manychskoe [Ponomarev et al., 2006].

MATERIAL. $20^{7} 0^{7}$, Apanasenkovskoe Distr., Manychskoe Vil., Manych-Gudilo Lake shore, 24-26.05.2005 (T.Kh.).

\section{ZODARIIDAE}

Zodarion morosum Denis, 1935

MATERIAL. $1 \sigma^{7}, 1$, $22 \mathrm{~km}$ NE of Arzgir Vil., reeds along Chograi Reservoir bank, 14-25.07.1987 (S.A.).

Zodarion rubidum Simon, 1914

Figs. 16, 17.

MATERIAL. $1 \sigma^{7}$, Izobilnyi, 9.07-8.08.2011 (V.K.); $1 \sigma^{\top}, 1$ ㅇ, s.1., 5.07-15.08.2014 (V.K.)

COMMENTS. This species is new to the spider fauna of Russia, known from Southern and Central Europe [Bosmans, 1997], as well as from the southeast of Ukraine [Ponomarev et al., 2016] and South Ossetia [Ponomarev, Komarov, 2015].

\section{Conclusions}

To date, the spider fauna of Stavropol Province, in its present administrative borders, comprises 265 species of 26 families (Table 1). The records of 154 species given here are new to Stavropol Province. One species (Zodarion rubidium) is new to the spider fauna of Russia.

The results presented in this paper are to be considered preliminary, because such families as Linyphiidae, Philodromidae, Salticidae, Theridiidae, Thomisidae and Titanoecidae are clearly under-represented in our samples. New findings are also expected in the family Gnaphosidae. Thus, it is safe to conclude that the spider fauna of Stavropol Province needs further investigations.
ACKNOWLEDGMENTS. We are sincerely thankful to S.V. Pushkin, T.V. Khanov, E. Khomchenko (Stavropol), A.A. Zotov, P.P. Ivliev, A.E. Rudaikov, E.N. Terskov (Rostov-on-Don), Yu.A. Manelov (Essentuki) for providing us with the material used in the present study. We are grateful to K.V. Dvadenko (Rostov-on-Don) for taking SEM and digital images presented in this paper, and also to I.V. Sheverdiaev and A.I Ermolaev (Rostov-on-Don) for the computer processing of these images. We wish to sincerely thank A.V. Tanasevich for his kind help with identifying Linyphiidae species. Finally, we would like to thank Sergey I. Tarasov (University of Tennessee, Knoxville: National Institute for Mathematical and Biological Synthesis) for translating the ms into the English.

This study was conducted within the frame of the Government plan IAZ SCC RAS, project \# 0259-2014-0005.

\section{References}

Bosmans R. 1997. Revision of the genus Zodarion Walckenaer, 1833, part II. Western and Central Europe, including Italy (Araneae: Zodariidae) // Bull. Br. Arachnol. Soc. Vol. 10. Pt. 8. P. 265-294.

Dunin P.M. 1992. The spider family Dysderidae of the Caucasian fauna (Arachnida Aranei Haplogynae) // Arthropoda Selecta. Vol.1. No.3. P.35-76.

Esyunin S.L., Tuneva T.K. 2009. [A review of Palaearctic lynxspiders of the heterophthalmus group of the genus Oxyopes (Aranei, Oxyopidae)] // Zoologicheskii zhurnal. Vol.88. No.2 P.164-175 [in Russian, with English summary].

Esyunin S.L., Tuneva T.K. 2012. [Two spider species (Araneae) new to fauna of Russia] // Vestnik Permskogo universiteta. Biologiya. No.2. P.36-37 [in Russian, with English summary].

Khanov T.V., Ponomarev A.V. 2006. [Spiders (Aranei) families Philodromidae and Thomisidae plains of Stavropol Province] // Caucasian Entomological Bulletin. Vol.2. No.1. P.14 [in Russian].

Köppen F.T. 1881. Über einige in Russland vorkommende giftige und vermeintlich giftige Spinnen // Beiträge z. Kenntnis d. Russischen Reiches. 2te Folge. St. Petersburg: Kais. Akad. Wiss. Bd.4. S.179-227.

Kovblyuk M.M. 2005. [Little-Known Species of Genus Zelotes (Aranei, Gnaphosidae) from Crimea] // Vestnik zoologii. Vol.39. No.5. P.3-14 [in Russian, with Englidh summary].

Kovblyuk N.M., Ponomarev A.V. 2008. [New and interesting spiders (Aranei: Agelenidae, Corinnidae, Gnaphosidae, Nemesiidae, Thomisidae) from the West Caucasus] // Caucasian Entomological Bulletin. Vol.4. No.2. P.143-154 [in Russian, with English summary].

Kovblyuk M.M., Tuneva T.K. 2009. Three interesting species of Gnaphosidae from Crimea (Arachnida: Aranei) // Arthropoda Selecta. Vol. 17 (for 2008). Nos.3-4. P.157-164.

Logunov D.V. 1998. Pseudeuophrys is a valid genus of the jumping spiders (Araneae, Salticidae) // Rev. Arachnol. T.12. Fasc.11. P.109-128.

Logunov D.V. 2015. Taxonomic-faunistic notes on the jumping spiders of the Mediterranean (Aranei: Salticidae) // Arthropoda Selecta. Vol.24. No.1. P.33-85.

Logunov D.V., Guseinov E.F. 2002. Faunistic review of the jumping spiders of Azerbaijan (Aranei: Salticidae), with additional faunistic records from neighbouring Caucasian countries // Arthropoda Selecta. Vol.10. No.3. P.243-260.

Logunov D.V., Penney D. 2004. A Post-Colloquium Arachnological Trip to Ciscaucasia, Russia // Newsl. Br. arachnol. Soc. No.99. P.4-5.

Lukianov N. 1897. [A list of spiders (Araneina, Pseudoscorpionina and Phalangina) living in the South-Western Province and 
adjacent gouvernements of Russia] // Zapiski Kievskogo obshchestva estestvoispytatelei. Vol.14. No.2. P.1-19 [in Russian].

Marusik Yu.M., Lehtinen P.T., Kovblyuk M.M. 2005. Cozyptila, a new genus of crab spiders (Aranei: Thomisidae: Thomisinae: Coriarachnini) from the western Palaearctic // Arthropoda Selecta. Vol.13 (for 2004). No.3. P.151-163.

Mikhailov K.G. 1987. Contribution to the spider fauna of the genus Micaria Westring, 1851 of the USSR. I (Aranei, Gnaphosidae) // Spixiana. Bd.10. H.3. P.319-334.

Mikhailov K.G. 1990. The spider genus Clubiona Latreille 1804 in the Caucasus, USSR (Arachnida: Araneae: Clubionidae) // Senckenberg. biol. Bd.70. H.4/6. S.299-322.

Mikhailov K.G. 1992. The spider genus Clubiona Latreille, 1804 (Arachnida Aranei Clubionidae in the USSR fauna: a critical review with taxonomical remarks // Arthropoda Selecta. Vol.1. No.3. P.3-34.

Mikhailov K.G. 2003. The spider genus Clubiona Latreille, 1804 (Aranei: Clubionidae) in the fauna of the former USSR: 2003 update // Arthropoda Selecta. Vol.11 (for 2002). No.4. P.283317.

Platnick N.I., Ovtsharenko V.I. 1991. On Eurasian and American Talanites (Araneae, Gnaphosidae) // J. Arachnol. Vol.19. No.2. P.115-121.

Platnick N.I., Song D.X. 1984. A Review oft he Zelotine Spiders (Araneae, Gnaphosidae) of China // Amer. Mus. Novitates. No.2848. P.1-22.

Ponomarev A.V. 2007. [New taxa of spiders (Aranei) from the south of Russia and Western Kasakhstan] // Caucasian Entomological Bulletin. Vol.3. No.2. P.87-95 [in Russian, with English summary].

Ponomarev A.V. 2008a. [The additional data to the spider fauna (Aranei) of the South-east of Russian Plain] // Vestnik Yuzhnogo nauchnogo tsentra RAN. Vol.4. No.3. P.78-86 [in Russian, with English summary].

Ponomarev A.V. 2008b. [Additions to fauna of spiders (Aranei) of the from south of Russia and Western Kazakhstan: new taxa and finds] // Caucasian Entomological Bulletin. Vol.4. No.1. P.49-61 [in Russian, with English summary].

Ponomarev A.V., Abdurakhmanov G.M., Alieva S.V., Dvadnenko K.V. 2011. [Spiders (Arachnida: Aranei) of the coastal and island territories of northern Dagestan] // Yugh Rossii: ekologiya, razvitie. No.4. P.126-143 [in Russian, with English summary].

Ponomarev A.V., Belosludtsev E.A., Dvadnenko K.V. 2008. [Spiders (Aranei) of the Lower Volga Region (Astrakhan and Volgograd areas of Russia) with the description of new taxa] // Caucasian Entomological Bulletin. Vol.4. No.2. P.163-185 [in Russian, with English summary].

Ponomarev A.V., Khanov T.V., Tsvetkov A.S. 2006. [Preliminary data on the fauna of spiders (Aranei) Stavropol Territory] // "Musei-zapovednik: ekologiya i kultura". Materialy vtoroi nauch.-prakt. konf. (stanitsa Veshenskaya, 13-16 sentyabrya 2006). Veshenskaya. P.246-247 [in Russian].

Ponomarev A.V., Komarov Yu.E. 2015. [Spiders (Aranei) of the Republic of South Ossetia] // Yugh Rossii: ekologiya, razvitie. Vol.10. No.1. P.116-147 [in Russian, with English summary]. Ponomarev A.V., Mikhailov K.G. 2007. [Addition to Fauna of spiders (Aranei) of the Russian Caucasus] // Lebedeva N.V. (ed.). Bioraznoobrasie i transformatsia gornykh ekosistem Kavkasa. Trudy Yuzhnogo nauchnogo tsentra RAN. T.3. Rostov-on-Don: YuNTs RAN. P.130-151 [in Russian].

Ponomarev A.V., Prokopenko E.V., Ivliev P.P., Shmatko V.Yu. 2016. [Spiders (Aranei) of the coast of Taganrog Bay (the Sea of Azov) and the Don River delta] // Caucasian Entomological Bulletin. Vol.12. No.1. P.3-28 [in Russian, with English summary].

Ponomarev A.V., Tsvetkov A.S. 2006. [New and rare spiders of family Gnaphosidae (Aranei) from a southeast of Europe] // Caucasian Entomological Bulletin. Vol.2. No.1. P.5-13 [in Russian, with English summary]

Schmidt P. 1895. Beitrag zur Kenntniss der Laufspinnen (Araneae Citigradae Thor.) Russlands // Zool. Jb., Abt. Syst. Bd.8. Nr.4. S.439-484.

Spassky S.A. 1936. [Spider species and genera new to the USSR fauna] // Trudy Zoologicheskogo instituta AN SSSR. T.3. P.533-536 [in Russian].

Szita E., Logunov D. 2008. A review of the histrio group of the spider genus Philodromus Walckenaer, 1826 (Araneae, Philodromidae) of the Eastern Palaearctic region // Acta Zoologica Academiae Scientiarum Hungaricae. Vol.54. No.1. P.23-73.

Tanasevitch A.V. 1987. The linyphiid spiders of the Caucasus, USSR (Arachnida: Araneae: Linyphiidae) // Senckenberg. biol. Bd.67. H.4-6. P.297-383.

Tanasevitch A.V. 1990. [Spiders of the family Linyphiidae of the Caucasian fauna (Arachnida, Aranei)] // Striganova B.R. (ed.). Fauna nasemnykh bespozvonochnykh Kavkaza. Moscow: Nauka Publ. P.5-114 [in Russian], 235 [English summary].

Titova E.V., Egorova N.S. 1978. [Evaluation of trophic relation of spiders with Eurygaster integriceps Put. (Heteroptera, Scutelleridae) by the serological method] // Entomologicheskoe obozrenie. T.57. No.2. P.284-289 [in Russian].

Verzhbitsky E. 1902. [On the spiders of the Caucasian Province] // Zapiski Kievskogo obshchestva estestvoispytatelei. Vol.17. No.2. P.461-504, tabl. VI [in Russian].

Zonshtein S.L. 1985. [Preliminary data on spiders of the suborder Mygalomorphae of the USSR fauna] // Fauna i ecologiya paukov SSSR. Trudy Zoologicheskogo instituta AN SSSR. Vol.139. P. 156-161 [in Russian].

Zyuzin A.A., Logunov D.V. 2000. New and little-known species of the Lycosidae from Azerbaijan, the Caucasus (Araneae, Lycosidae) // Bull. Br. arachnol. Soc. Vol.11. Pt.8. P.305-319.

Responsible editors D.V.Logunov, K.G. Mikhailov 